ISSN 2079-9292

www.mdpi.com/journal/electronics

Review

\title{
Two-Dimensional Materials for Sensing: Graphene and Beyond
}

\author{
Seba Sara Varghese ${ }^{1,2}$, Saino Hanna Varghese ${ }^{3}$, Sundaram Swaminathan ${ }^{2}$, \\ Krishna Kumar Singh ${ }^{4}$ and Vikas Mittal ${ }^{1, *}$
}

1 Department of Chemical Engineering, The Petroleum Institute, Abu Dhabi 2533, United Arab Emirates; E-Mail: sebavarghese@gmail.com

2 Department of Electrical and Electronics Engineering, Birla Institute of Technology and Science Pilani, Dubai Campus, Dubai 345055, United Arab Emirates;

E-Mail: swami@dubai.bits-pilani.ac.in

3 Department of Chemistry, Catholicate College, Pathanamthitta, 689645 Kerala, India; E-Mail: sainoshiju@gmail.com

4 Department of Physics, Birla Institute of Technology and Science Pilani, Dubai Campus, Dubai 345055, United Arab Emirates; E-Mail: singh@dubai.bits-pilani.ac.in

* Author to whom correspondence should be addressed; E-Mail: vmittal@pi.ac.ae; Tel.: +971-2-607-5491.

Academic Editor: Frank Schwierz

Received: 3 August 2015 / Accepted: 14 September 2015 / Published: 18 September 2015

\begin{abstract}
Two-dimensional materials have attracted great scientific attention due to their unusual and fascinating properties for use in electronics, spintronics, photovoltaics, medicine, composites, etc. Graphene, transition metal dichalcogenides such as $\mathrm{MoS}_{2}$, phosphorene, etc., which belong to the family of two-dimensional materials, have shown great promise for gas sensing applications due to their high surface-to-volume ratio, low noise and sensitivity of electronic properties to the changes in the surroundings. Two-dimensional nanostructured semiconducting metal oxide based gas sensors have also been recognized as successful gas detection devices. This review aims to provide the latest advancements in the field of gas sensors based on various two-dimensional materials with the main focus on sensor performance metrics such as sensitivity, specificity, detection limit, response time, and reversibility. Both experimental and theoretical studies on the gas sensing properties of graphene and other two-dimensional materials beyond graphene are also discussed. The article concludes with the current challenges and future prospects for two-dimensional materials in gas sensor applications.
\end{abstract}


Keywords: two-dimensional materials; gas sensing; electronic properties; sensor performance

\section{Introduction}

Detection of gas molecules is extremely important in environmental monitoring, industrial chemical processing, public safety, agriculture, medicine and indoor air quality control. For a long time, metal oxide semiconductor gas sensors have played an inevitable role in environmental contaminant detection and industrial process control [1]. Metal oxide semiconductors are the most widely used gas sensing materials due to their numerous advantages such as high sensitivity towards various gases with ease of fabrication, high compatibility with other processes, low cost, simplicity in measurements along with minimal power consumption [2-4]. The high operating temperatures $\left(200{ }^{\circ} \mathrm{C}\right.$ to $500{ }^{\circ} \mathrm{C}$ ) [5], long recovery periods, limited maximum sensitivity (in the range of parts-per-million), low specificity, and limited measurement accuracy [6] basically limited their applications in rapidly changing environment.

The increasing demand for highly sensitive, selective, cost-effective, low power consuming, stable and portable sensors has stimulated extensive research on new sensing materials. One of the most important features of a material to be used for gas sensing is its high surface-to-volume ratio. Nanostructures possess high surface-to-volume ratio which provides large active surface area for the interactions of gas molecules. This strongly favors the adsorption of gases on nanostructures and ultimately leads to highly sensitive sensor performance [7]. One-dimensional (1D) nanostructures are particularly suited for gas detection applications because of their large surface-to-volume ratio, good thermal and chemical stabilities, and sensitivity of electrical properties to changes in the surroundings [8]. Gas sensors using 1D nanostructures such as carbon nanotubes (CNTs) [9-12] and nanowires (NWs) [4,13-15] have demonstrated excellent performance for gas sensing [16,17] with high sensitivity down to parts-per-billion (ppb) levels [18], fast response (time scale in the order of seconds) [19], good selectivity along with low power consumption and miniaturization.

Two-dimensional (2D) materials have captured the interest of research community after the first successful isolation of graphene sheets by micromechanical exfoliation of highly-oriented pyrolytic graphite in 2004 [20]. Graphene, a one-atom-thick sheet of carbon atoms with a 2D hexagonal crystal structure, has shown great promise for applications such as electronics and photonics [21-24], energy conversion and storage [25-29], medicine [30-34], chemical and biological sensing [35-38], etc., due to its interesting physical, chemical, electrical, optical, thermal and mechanical properties [39-42]. Many experimental and theoretical reports on gas sensors made from graphene [43-57] and its derivatives such as graphene oxide (GO) [58-61], reduced graphene oxide (rGO) [62-66] proved that graphene and its derivatives could be used as efficient sensing materials for next-generation gas sensing systems. Inspired by the superior performance of the first 2D material, graphene for gas sensing and many other applications, a lot of research effort has been devoted to the isolation of other 2D materials which exist as strongly bonded stacked layers in bulk crystals and their potential applications. So far, several hundreds of different 2D materials with extraordinary properties are known which include allotropes of various elements such as graphyne, silicene, germanene, 
phosphorene. etc., and compounds such as transition metal dichalcogenides (TMDs) (for example, $\mathrm{MoS}_{2}, \mathrm{MoSe} 2, \mathrm{WTe} 2, \mathrm{TaS}_{2}, \mathrm{TaSe}_{2}$, etc.), germanane, hexagonal boron nitride (BN), etc. [67]. These family of materials have good optical transparency, excellent mechanical flexibility, great mechanical strength and also peculiar electrical properties, which greatly favors their applications in electronics, optoelectronics [68,69], etc. 2D materials provide a promising platform for the development of ultrahigh sensitive and highly selective sensors by tailoring their rich surface chemistry without any deterioration of their unique optical and electrical properties.

So far, many review articles on 2D materials discussing their novel physical, electronic properties, recent developments in synthesis techniques and their applications in electronics, optoelectronics, energy conversion and storage, gas storage, etc., have been published [67,70-75]. During the past few years, there have been some comprehensive reviews on gas sensors based on graphene and graphene related materials with primary focus on the properties for gas sensing, sensing mechanisms, modifications of graphene for enhanced sensing features, etc. [76-78]. To the best of our knowledge, no specific review on the role of two-dimensional materials in gas sensing has been reported. This necessitates a summary of the latest developments in the area of gas sensors by employing graphene and other 2D materials beyond graphene as sensing materials, with emphasis on the sensing performance indicators such as sensitivity, selectivity, detection threshold, response and recovery times. The novel properties of 2D materials that make them perfectly suitable as sensing elements for gas sensor systems are described. In addition to experimental verifications of 2D material based gas sensors, theoretical first-principles studies on the adsorption of various gas molecules on $2 \mathrm{D}$ materials are also discussed.

\section{Two-Dimensional Materials for Gas Sensing}

2D materials are basically single layer materials with thickness of few nanometers or even less. The high surface area of $2 \mathrm{D}$ materials provides large number of reactive sites, which make these materials efficient in sensing, catalysis and energy storage technologies. In addition to large surface area, the high electrical conductivities and low noise of the $2 \mathrm{D}$ materials also contribute to their indispensable role in future gas detection systems. Due to low electrical noise and high electrical conductivity, a small change in carrier concentration induced by gas exposure could lead to significant changes in electrical conductivity. These features along with the charge transfer between gas molecules and these materials associated with the adsorption of gas molecules make these materials one of the best suitable candidates for the fabrication of gas sensors [79].

The discovery of graphene and understanding of their properties encouraged scientists to investigate the potential applications of graphene and graphene based materials as gas sensing materials [44]. The utilization of graphene as an ultrasensitive sensing element had emerged as one of the most significant applications of graphene. The sensing property of graphene could be attributed to its two dimensional structure which provides advantages such as: (i) Maximum surface area per unit volume ratio and (ii) High sensitivity of electron transport through graphene to the adsorption of gas molecules [36]. The astonishing gas sensing performance of graphene with a 2D structure inspired researchers to investigate other $2 \mathrm{D}$ materials, which were discovered after graphene for their gas sensing characteristics. All 2D materials which include graphene, $\mathrm{MoS}_{2}$, phosphorene, etc., rely on the 
change in electrical conductivity upon their interaction with gaseous species due to the induced charge transfer by the gas molecules present in the surroundings. Besides graphene, research has also centered on gas sensors based on various 2D materials such as TMDs especially $\mathrm{MoS}_{2}$ [80-87], $\mathrm{WS}_{2}$ [88-90], $\mathrm{MoSe}_{2}$ [91] and phosphorene [92,93]. 2D nanostructures in the form of nanosheets (NSs), nanowalls, nanoplates, etc., made from $\mathrm{ZnO}, \mathrm{NiO}, \mathrm{CuO}, \mathrm{WO}_{3}, \mathrm{SnO}_{2}$, etc., have also proven as successful sensing materials, which could be used as building blocks for the fabrication of gas sensors [94-108].

\subsection{Graphene}

Graphene, the basic building block of other carbon based allotropes such as graphite, CNTs and fullerenes, was first isolated by Geim and Novoselov of the University of Manchester, for which they were awarded the Nobel Prize in Physics in 2010. Since then, the study of this material has become one of the hottest topics for material scientists due to the exotic thermal, optical, electrical, mechanical and physiochemical properties arising from the two-dimensional crystal structure [20]. The interesting properties of graphene that have been explored so far include linear energy-dispersion at the Dirac point, existence of massless relativistic particles at the Dirac point, quantum Hall-effect at room temperature, exceptionally high charge carrier mobility at room temperature, high electrical and thermal conductivity, great mechanical strength, high optical transparency and high specific surface area $[21,39,109-113]$. These unique characteristics make graphene a suitable material for a vast variety of applications.

Soon after the discovery of graphene and graphene oxide by mechanical and chemical exfoliation of 3D bulk graphite, scientists started analyzing the usefulness of graphene and its derivatives for gas sensing. 2D-graphene possess large surface area compared to 1D-CNTs due to the fact that every carbon atom in graphene acts as an active site for the gas molecules present in the surroundings to interact with. In addition to this, electrical properties of graphene such as high charge carrier mobilities at room temperature [109], metallic conductivity and low Johnson noise [21,79] also contribute to the rise of graphene as one of the best suitable materials for gas sensing [44]. The defect less high quality crystal structure of graphene being a $2 \mathrm{D}$ material screens the $(1 / \mathrm{f})$ noise caused by thermal switching better than other 1D and 0D structures [114].

Graphene based gas sensors work on the principle that introduction of gas molecules change the local charge carrier concentration in graphene by either increasing or decreasing the concentration of electrons (as shown in Figure 1a) depending on the nature of gas species (electron donor or acceptor) which leads to corresponding decrease or increase in electrical conductivity [43-45]. Graphene possess the capability to induce noticeable changes in the electronic properties even by a small change in the carrier concentration in graphene by the gas adsorbates due to high signal-to-noise ratio contributed by its features [44].

Several research groups used pristine graphene for sensing gas molecules such as $\mathrm{CO}_{2}, \mathrm{NH}_{3}, \mathrm{NO}_{2}$, $\mathrm{NO}, \mathrm{N}_{2} \mathrm{O}, \mathrm{O}_{2}, \mathrm{SO}_{2}, \mathrm{H}_{2} \mathrm{O}$, etc., [47-56] since Schedin et al. [44] demonstrated the first micrometer-sized sensor made from graphene that was capable of detecting gases at the ultimate concentration (single molecule level) at room temperature. This sensor responded rapidly to the attachment and the detachment of a single $\mathrm{NO}_{2}$ gas molecule from graphene's surface. The sensitivity reported for the first graphene sensor was nearly several orders of magnitude greater than that of the 
previous sensors. The observed changes in the resistivity curve reflected the type of the gas species, either electron donor or acceptor. Out of the considered gases, the adsorption of $\mathrm{NO}_{2}$ and $\mathrm{H}_{2} \mathrm{O}$ led to decrease in resistivity which indicated their electron acceptor nature, whereas the adsorption of $\mathrm{NH}_{3}$ and $\mathrm{CO}$ led to increase in resistivity which indicated their electron donor nature as shown in Figure $1 \mathrm{~b}$. Even after stopping the gas flow, the sensor recovery was achieved only after annealing the device at $150{ }^{\circ} \mathrm{C}$ (as shown in Section IV of Figure 1b).
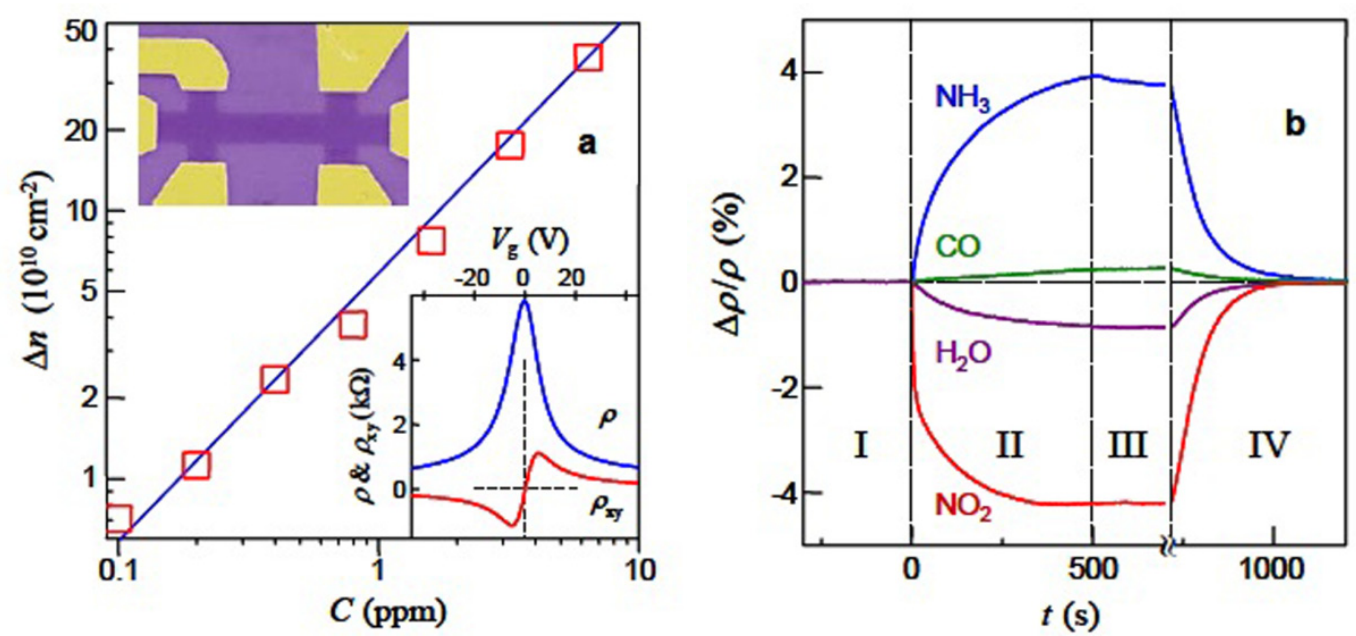

Figure 1. Sensitivity of graphene to chemical doping. (a) Concentration $\Delta n$ of chemically-induced charge carriers in single-layer graphene exposed to different concentrations of $\mathrm{NO}_{2}$. Upper inset: scanning-electron micrograph of this device. The scale of the micrograph is given by the width of the Hall bar, which is $1 \mu \mathrm{m}$. Lower inset: Characterization of the graphene device by using the electric field effect; (b) Changes in resistivity $\rho$ caused by graphene's exposure to various gases diluted in concentration $1 \mathrm{ppm}$. The positive (negative) sign of changes is chosen here to indicate electron (hole) doping. Region I-the device is in vacuum prior to its exposure; II-exposure to a 5 liter volume of a diluted chemical; III-evacuation of the experimental setup; and IV_-annealing at $150{ }^{\circ} \mathrm{C}$. Reprinted with permission from Ref. [44]. Copyright, 2007, Nature Publishing Company Ltd.

Chen et al. [52] demonstrated the detection of a wide range of gas molecules using pristine graphene synthesized by chemical vapor deposition (CVD) at very low concentrations. Detection limit (DL) as low as 158 parts-per-quadrillion (ppq) to NO gas molecules with a signal-to-noise ratio of 3 was achieved at room temperature under inert atmosphere by continuous in situ cleaning of graphene's surface by ultraviolet (UV) light illumination. The sensor also showed fast response, good reproducibility and also $80 \%$ of recovery within few minutes of exposure to a mixture of 10 parts-per-trillion (ppt) NO in $\mathrm{N}_{2}$. The obtained sensitivity was nearly $300 \%$ better than similar CNT based sensors and also the DLs were lower than the lowest detection levels reported for other nanosensors [52].

Low-frequency electronic noise spectrum was employed as a sensing parameter to improve the selectivity of graphene to vapors of different chemicals [51]. They observed that the vapors of all considered chemicals (chloroform, methanol, tetrohydrofuran, acetonitrile, ethanol, methylene chloride 
and toluene) change the electrical resistance of graphene sensors. But only chloroform, methanol, tetrohydrofuran, acetonitrile and ethanol changed the noise spectrum of single layer graphene transistors by inducing Lorentzian components with different characteristic frequencies, whereas toluene and methylene chloride do not modify the noise spectrum. Rumyantsev and his group proved that a single pristine graphene device could achieve good selectivity along with high sensitivity by combining low-frequency noise spectrum measurements along with the change in resistance [51].

Electrochemically exfoliated few layered graphene (FLG, 3- to 10-layer graphene) exhibited good $\mathrm{CO}_{2}$ and liquid petroleum gas (LPG) sensing performance such as high sensitivity (3.83 and 0.92 for $\mathrm{CO}_{2}$ and LPG respectively), response time (11 s and $5 \mathrm{~s}$ for $\mathrm{CO}_{2}$ and LPG respectively), and recovery time (14 s and $8 \mathrm{~s}$ for $\mathrm{CO}_{2}$ and LPG respectively) at operating temperatures of $423 \mathrm{~K}$ and $398 \mathrm{~K}$ respectively [54]. The detection limit of the chemiresistive graphene based sensor was found to be 3 ppm and 4 ppm for $\mathrm{CO}_{2}$ and LPG respectively with excellent stability at room temperature [54]. The good LPG sensing behavior of the FLG based chemiresistive gas sensor at relatively low temperatures promise their use for practical LPG detection.

The huge cost associated with the fabrication of graphene based devices remains as a great obstacle for widespread gas sensing using graphene. Recently, a simple, low power, low cost resistive gas sensor based on graphene-paper (G-paper) prepared by direct transfer of graphene layers on to paper (as shown in Figure 2a) without any intermediate layers was demonstrated for the first time by Kumar et al. [55]. The achieved resolution limit of $~ 300$ ppt was better than other sensors based on graphitic and semiconducting metal oxides using paper as substrate and these sensors were capable of withstanding minor strain. The G-paper strip showed $\sim 65 \%$ increase in conductance in $1400 \mathrm{~s}$ to the flow of $2.5 \mathrm{ppm} \mathrm{NO} 2$ gas and the conductance decrease by $\sim 15 \%$ in $1500 \mathrm{~s}$ on stopping the $\mathrm{NO}_{2}$ flow (as shown in Figure 2b). Cleaning of graphene by UV exposure to remove the adsorbed gas molecules dramatically reduced the recovery time (from hours to $30 \mathrm{~s}$ ), but the overall response and the characteristic time constant got improved.

Ricciardella et al. [56] demonstrated the potential of inkjet printing technique for manufacturing chemi-resistive sensors based on liquid phase exfoliated (LPE) graphene. This technique allows deposition of small ink volumes with a more controlled drying process, which ensures good printed film quality compared to drop casting method. The LPE graphene being a p-type material, showed a decrease in current on exposure to $\mathrm{NH}_{3}$ (electron donor) and an increase in current on $\mathrm{NO}_{2}$ exposure (electron acceptor), thus enabling specific detection of these gases. The fabricated sensor exhibited good repeatability upon exposure to both gases at room temperature and atmospheric pressure with relative humidity of $50 \%$, which was found to be independent on the number of printed layers. The perennial issue of graphene based gas sensors operating in environmental conditions such as low reproducibility could be overcome by using inkjet printing technology for sensor fabrication [56]. 


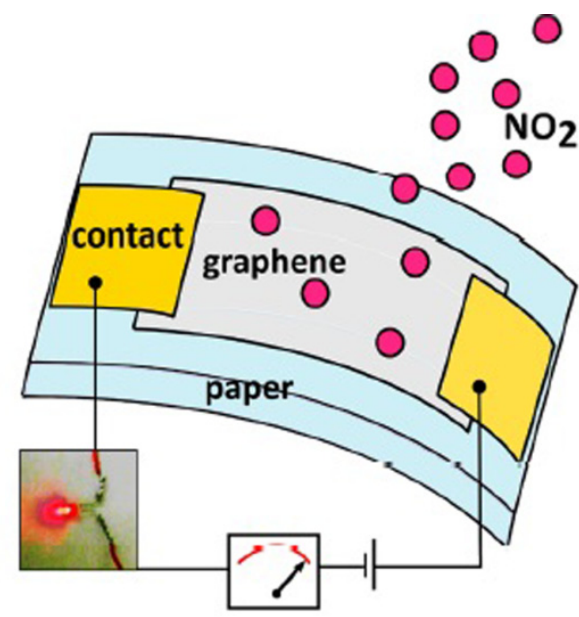

(a)

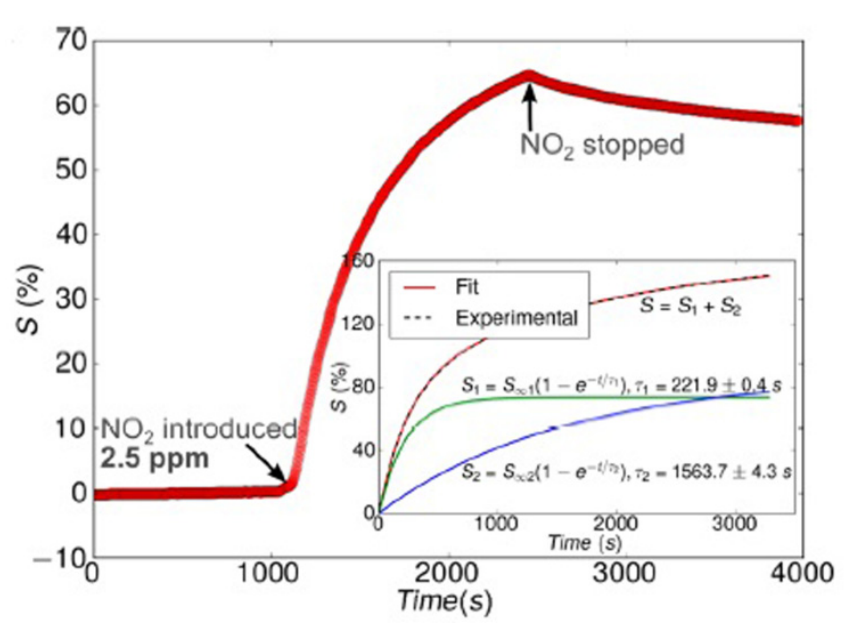

(b)

Figure 2. (a) Schematic of a G-paper strip based gas sensor; (b) Response of a G-paper strip to $2.5 \mathrm{ppm}$ of $\mathrm{NO}_{2}$. Inset shows a fit of double exponential function to the temporal response for $2.5 \mathrm{ppm}$ of $\mathrm{NO}_{2}$. The two constituent exponentials are also shown along with the estimates of time-constants. Reprinted with permission from Ref. [55]. Copyright, 2015, American Chemical Society.

Apart from experimental studies, theoreticians investigated the adsorption of gas molecules on pristine graphene to understand the interactions of graphene with gas molecules so as to fully exploit the potential of graphene for gas sensing. All theoretical works on pristine graphene using ab initio simulations based on quantum mechanics showed weak adsorption of gas molecules on its surface due to the highly stable and strongly bonded carbon atoms in graphene. The capability of graphene to sense gas molecules is usually investigated by inferring the results from the calculations of adsorption energies, charge transfers and the density of states of graphene-gas molecule adsorption systems $[43,45-57]$.

Using first-principles calculations based on density functional theory (DFT), it was found that gas molecules such as $\mathrm{H}_{2} \mathrm{O}, \mathrm{NH}_{3}, \mathrm{CO}, \mathrm{NO}_{2}$ and $\mathrm{NO}$ are only physically adsorbed on pristine graphene. The electron donating behavior of $\mathrm{CO}$ and $\mathrm{NH}_{3}$ and the electron withdrawing behavior of $\mathrm{NO}_{2}$ and $\mathrm{H}_{2} \mathrm{O}$ demonstrated experimentally was confirmed by simulations done on a graphene model [45]. For the physisorption of hydrogen on graphene flakes, first-principles non-local van der Waals (vdW) density functional (B3LYP-D3) method calculated an adsorption energy of $5.013\left(\mathrm{~kJ} \cdot \mathrm{mol}^{-1}\right)$, that was found to be in great agreement with the experimental results [57]. All these reports of gas adsorption on pristine graphene showed low adsorption energies due to the inert property of graphene, which makes it unsuitable for practical use.

Graphene oxide, single- or few layered-graphite oxide sheets obtained by chemical oxidation of graphite and subsequent exfoliation in water, are rich in oxygen-containing groups such as epoxies, hydroxyls, carboxyls, etc., on its basal planes and edges which makes it a versatile material for gas detection [58-61]. Prezioso and his co-workers [58] proposed a practical and highly sensitive gas sensor based on large and highly oxidized GO flakes prepared by modified Hummers' method. The sensor device was fabricated by drop casting GO monolayers with an average size of $\sim 30 \mu \mathrm{m}$ (about 2 orders of magnitude higher than that of commercially available GO) on platinum interdigitated 
electrodes. They measured a DL of 20 parts-per-billion (ppb) to $\mathrm{NO}_{2}$, which is the lowest value ever reported with other graphene based gas sensors. Compared to CNTs and reduced GO based sensors, the large number of active sites on GO lead to highly improved sensitivity and hence suited for applications that require high sensitivity but at the cost of slow response. GO has proved to be the best suitable material for humidity sensing applications with ultrahigh sensitivity [59]. Microscale capacitive humidity sensor fabricated using GO films exhibited excellent sensing properties such as a sensitivity of up to $37,800 \%$, which is more than 10 times greater than that of the best capacitive humidity sensors at $15 \%-95 \%$ relative humidity, fast response and fast recovery which are less than $1 / 4$ th and $1 / 2$ th of that of the conventional sensors respectively [59].

Few theoretical studies of gas molecular adsorption on GO have also been reported [60,61]. The adsorption of ammonia on graphene and graphene oxide investigated using first-principles calculations showed that the surface active sites such as epoxy and hydroxyls on GO surface enhanced the interactions between $\mathrm{NH}_{3}$ and $\mathrm{GO}$. The adsorption energy of $\mathrm{NH}_{3}$ on $\mathrm{GO}$ was found to be greater than that on pristine graphene due to the presence of hydroxyl and epoxy groups on graphene surface [60].

One of the most popular approach for the synthesis of graphene by thermal $[115,116]$ or chemical reduction $[117,118]$ of GO results in reduced GO (rGO), having almost identical structure of pristine graphene, but with several residual oxygen functional groups. $\mathrm{rGO}$ has also found extensive use in gas sensing [62-66] which could be attributed to the presence of some chemically active defect sites even after reduction of GO, high conductivity, capability for surface modification and water dispersibility. Ppb level detection of acetone and other toxic chemicals has been successfully demonstrated by a GO based chemical sensor reduced by hydrazine vapor. They observed that the level of GO reduction dependent on the exposure time to hydrazine vapor influenced the sensing response and the $1 / \mathrm{f}$ noise [62]. A 360\% increase in sensing response to $\mathrm{NO}_{2}$ gas at room temperature and atmospheric pressure was achieved by GO based field effect transistor (FET) gas sensors upon chemical reduction, on comparison with thermally reduced GO based sensors [63]. Even though rGO had demonstrated as a promising gas sensing material, selective detection of gas molecules remains as a great challenge for enabling practical use, similar to graphene. In this direction, Lipatov et al. fabricated a rGO based gas sensing system that could recognize different alcohols such as ethanol, methanol and isopropanol by making use of the significantly different properties of rGO flakes obtained from the same batch fabrication [64].

Later both experimental and theoretical studies proved that the sensitivity of graphene based gas sensors can be enhanced significantly by introducing dopants such as boron, nitrogen, phosphorus, gallium, chromium, manganese, silicon, sulphur, etc., and defects [119-124]. The introduction of dopants into the crystal structure and defects into the basal plane modifies the physical, chemical and electrical properties of graphene, that could be tailored for improving the sensitivity and selectivity of graphene based gas sensors [125]. Heteroatoms create new active sites on graphene surface that enables strong adsorption of gas molecules which has been proved by theoretical investigations of gas sensing using doped graphene. The strong chemical doping of doped graphene by the gas molecules was evident from the large values of adsorption energies and the charge transfers calculated using $a b$ initio calculations. Chromium and manganese are found to be the best suitable dopants for the sensitive detection of $\mathrm{SO}_{2}$ [120]. Zhang et al. proved strong chemisorption of $\mathrm{H}_{2} \mathrm{~S}$ on Fe-doped graphene, suggesting the possibility of employing Fe-doped graphene for $\mathrm{H}_{2} \mathrm{~S}$ gas detection [121] 
It has been found that nitrogen-doped graphene is the best material for selective sensing of CO [122]. First-principles study of the effect of the modification of graphene with Stones-Wales (SW) defect, Al doping and the combination of two on the $\mathrm{SO}_{2}$ adsorption behavior of graphene showed high chemical reactivity of Al-doped graphene and Al-doped graphene with $\mathrm{SW}$ defect towards $\mathrm{SO}_{2}$ compared to pristine graphene and SW-defected graphene [123]. Vacancy defected graphene along with dopants such as boron, nitrogen and sulphur was found to be more sensitive to formaldehyde than pristine graphene due to the strong chemisorption of formaldehyde on vacancy-defected graphene with dopants [124].

It was also proved experimentally that modification of graphene by dopant atoms enhances the gas sensing properties of graphene [126,127]. Niu et al. [127] reported excellent $\mathrm{NO}_{2}$ sensing ability of $\mathrm{N}$ and $\mathrm{Si}$ co-doped graphene nanosheets (NSi-GNS) prepared by high-temperature annealing of $\mathrm{N}$ and Si-containing graphene oxide-ionic liquid (GO-IL) composites (as shown in Figure 3). In the NSi-GNS, $\mathrm{N}$ atoms act as active sites for $\mathrm{NO}_{2}$ gas adsorption, whereas Si atoms modify the electronic structure significantly. NSi-GNS based gas sensor obtained by annealing of GO-IL at $400{ }^{\circ} \mathrm{C}$ (NSi-GNS-400) spread on a glass substrate between the silver electrodes (as shown in Figure 4a), showed high negative sensor response $(-26 \% \pm 1 \%)$ in $21 \mathrm{ppm}$ of $\mathrm{NO}_{2}$ and good stability even after five sensing cycles (as shown in Figure $4 \mathrm{~b}$ ). The response time and the recovery times observed for NSi-GNS-400 were $68 \mathrm{~s}$ and $635 \mathrm{~s}$ respectively (as shown in Figure 4c). The response of the NSi-GNS-400 upon $\mathrm{NO}_{2}$ exposure with concentrations varying from 21 to 1 ppm (as shown in Figure 4d) had a decreasing trend of response with decrease in $\mathrm{NO}_{2}$ concentration. The high sensitivity of the sensor was evident from the response value of $-8.8 \%$ at $1 \mathrm{ppm} \mathrm{NO}_{2}$ [127].

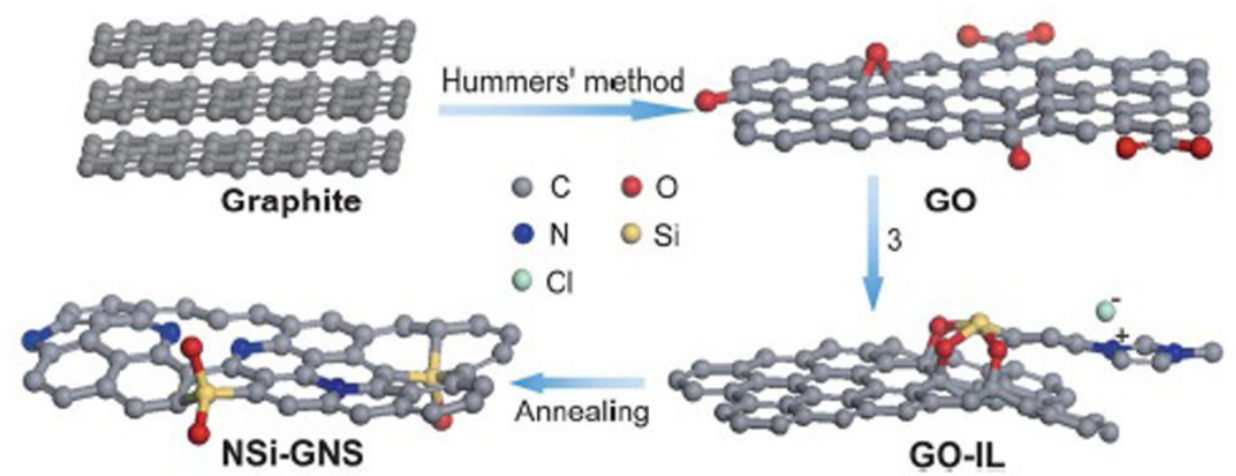

Figure 3. Schematic illustration of the synthesis of $\mathrm{N}$ and $\mathrm{Si}$ co-doped graphene nanosheets (NSi-GNS) through the high-temperature annealing of $\mathrm{N}$ and Si-containing graphene oxide-ionic liquid composite (GO-IL). Reprinted with permission from Ref. [127]. Copyright, 2013, Royal Society of Chemistry. 

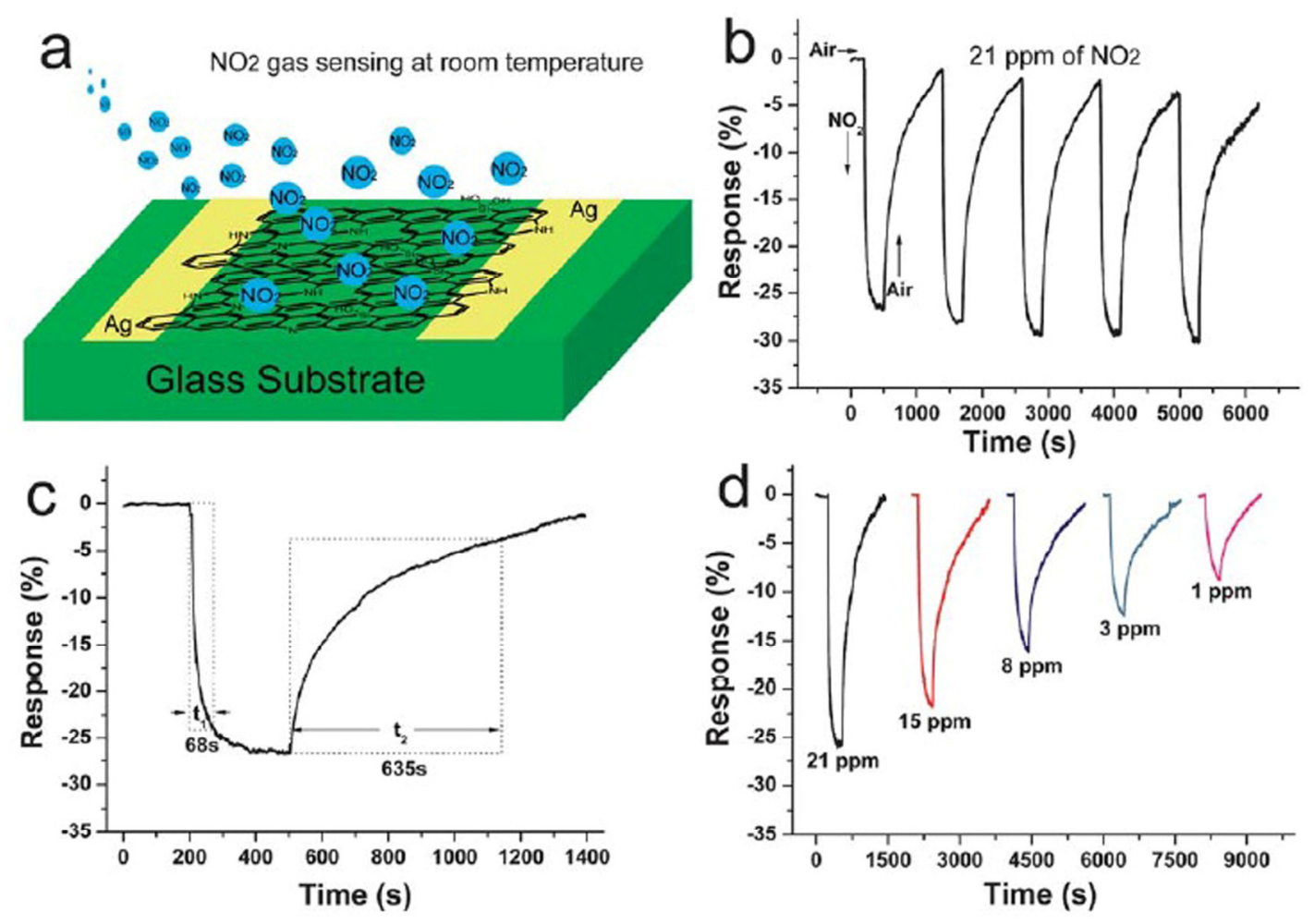

Figure 4. (a) $\mathrm{NO}_{2}$ sensor; (b) the response of NSi-GNS-400 to $21 \mathrm{ppm}$ of $\mathrm{NO}_{2}$; (c) response and recovery time; and (d) response to $\mathrm{NO}_{2}$ with varying concentrations. Reprinted with permission from Ref. [127]. Copyright, 2013, Royal Society of Chemistry.

Apart from dopants and defects, other chemical functionalization methods such as modification with metal and metal oxide nanoparticles (NPs) and polymers have also been studied. Compared to pristine graphene and rGO, sensors based on graphene/rGO modified with nanoparticles of metals or metal oxides have demonstrated highly sensitive and selective sensing behavior [128-135], which could be attributed to large changes in the electronic properties of graphene/rGO upon gas exposure due to the synergistic effects of NPs and graphene/rGO.

Many reports in the literature prove that pristine graphene has poor sensitivity towards hydrogen gas owing to the absence of dangling bonds in the structure. Palladium (Pd) NP decorated CVD grown graphene showed good sensing response of $33 \%$ at room temperature to $1000 \mathrm{ppm} \mathrm{H}_{2}$ with a detection limit of $20 \mathrm{ppm}$, due to the significant increase in resistance of the Pd decorated graphene sensor upon hydrogen injection. The sensor was found to be flexible that no significant degradation in the sensing response upon bending the sensor to a curved geometry [128].

Cho and his co-workers proved that the introduction of aluminium (Al) NPs and Pd NPs on graphene lead to improved sensitivity of graphene to $\mathrm{NO}_{2}$ and $\mathrm{NH}_{3}$ gases respectively [129]. Wang et al. [130] fabricated gas sensors based on rGO functionalized with platinum (Pt) NPs by mid-temperature thermal annealing and alternating current dielectrophoretic technique, which allowed for efficient sensing of multiple gases. At room temperature, the rGO gas sensors with (without) $\mathrm{Pt}$ NPs exhibited sensitivities of $14 \%(7 \%), 8 \%(5 \%)$, and $10 \%(8 \%)$, for $1000 \mathrm{ppm} \mathrm{H}_{2}, \mathrm{NH}_{3}$, and $\mathrm{NO}$ gases, respectively. An improvement of $100 \%, 60 \%$ and $25 \%$, to $\mathrm{H}_{2}, \mathrm{NH}_{3}$, and $\mathrm{NO}$ gases was observed for $\mathrm{Pt}$ functionalized $\mathrm{rGO}$ compared to that of $\mathrm{rGO}$ sensors without $\mathrm{Pt} \mathrm{NP}$ decoration. 
The recovery/response time for $\mathrm{H}_{2}$ gas was found to decrease with Pt decoration while for $\mathrm{NH}_{3}$, and NO, it showed the opposite behavior [130].

Recently, gas sensors based on graphene transistors decorated with $\mathrm{SnO}_{2}$ NPs exhibited high selectivity, fast response and short recovery $\left(\sim 1\right.$ s) to $100 \mathrm{ppm} \mathrm{H}_{2}$ at $50{ }^{\circ} \mathrm{C}$ (as shown in Figure 5a-c) [131]. FETs based on graphene decorated with metal oxide NPs were employed for developing high performance hydrogen gas sensors. The extremely high surface-to-volume ratio and the abundance of dangling bonds by the decoration of graphene with metal oxide NPs, resulted in strong interactions of gas molecules in the surroundings through the grain boundaries and unsaturated bonds of metal oxide NPs on graphene (as shown in Figure 6b), which ultimately resulted in excellent $\mathrm{H}_{2}$ sensitivity of the graphene-metal oxide NP hybrid, compared to pristine graphene (as shown in Figure 6a). Graphene facilitates quick transfer of electrons from metal oxide NPs due to the insignificant Schottky barrier caused by the matching work functions of NPs and graphene. They observed an increase in output current with increase in $\mathrm{H}_{2}$ concentration from $1 \mathrm{ppm}$ to $100 \mathrm{ppm}$ with good reproducibility (as shown in Figure 5b). The achieved lowest resolution limit of $1 \mathrm{ppm}$ proves the potential of $\mathrm{SnO}_{2} \mathrm{NP}$-graphene for high sensitive low level detection of $\mathrm{H}_{2}$ gas [131].

$\mathrm{rGO} / \mathrm{ZnO}$ nanocomposites have recently been employed as highly sensitive $\mathrm{NO}_{2}$ gas sensors at room temperature with fast response and recovery than those based on pristine rGO, which clearly indicate the improvement in the sensing property of rGO by $\mathrm{ZnO}$ NP decoration due to the tuning of the semiconducting properties of $\mathrm{rGO}$ induced by $\mathrm{ZnO}$ [132]. Apart from $\mathrm{SnO}_{2}$ and $\mathrm{ZnO}$, graphene/rGO decorated with other metal oxides such as $\mathrm{Cu}_{2} \mathrm{O}$ [133], $\mathrm{WO}_{3}$ [134], $\mathrm{Fe}_{2} \mathrm{O}_{3}$ [135], etc., have also demonstrated to be successful candidates for the detection of various gases due to the higher sensing response as compared to non-decorated graphene/rGO.

(a)

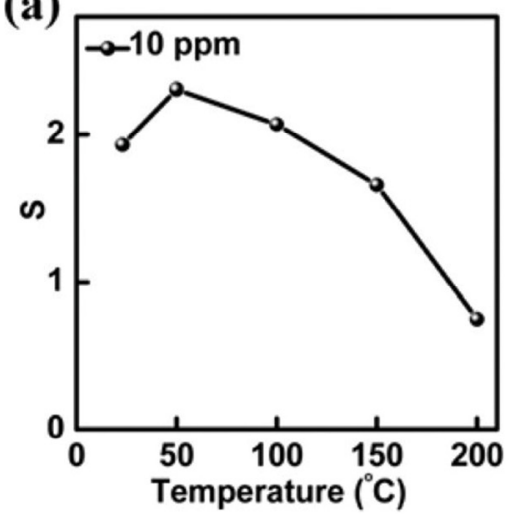

(b)

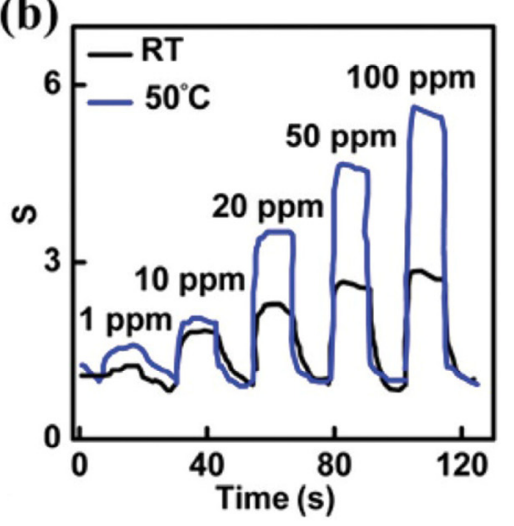

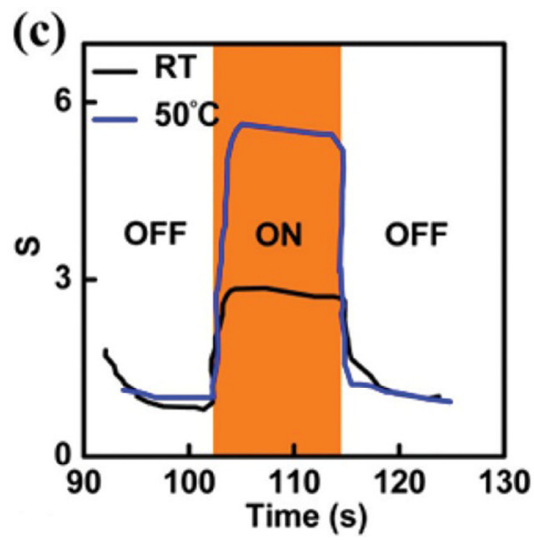

Figure 5. (a) The sensitivity of the gas sensor decorated with $\mathrm{SnO}_{2}$ nanoparticles (NPs) at various temperatures; (b) Real-time dynamic response of gas sensors decorated with $\mathrm{SnO}_{2}$ NPs exposed to different $\mathrm{H}_{2}$ concentrations at different operation temperatures; (c) The response and recovery times of the sensor exposed to the $100 \mathrm{ppm} \mathrm{H}_{2}$ concentration. Reprinted with permission from Ref. [131]. Copyright, 2015, Royal Society of Chemistry. 
(a)

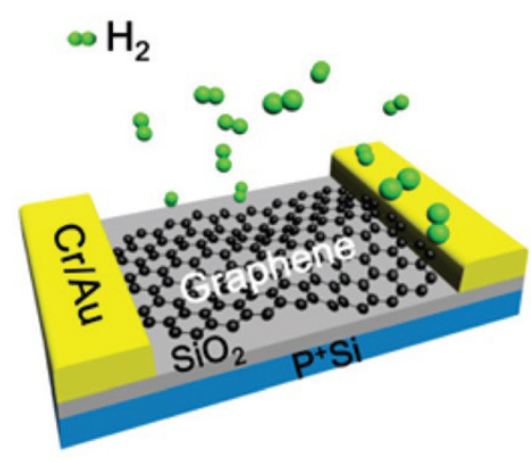

(b)

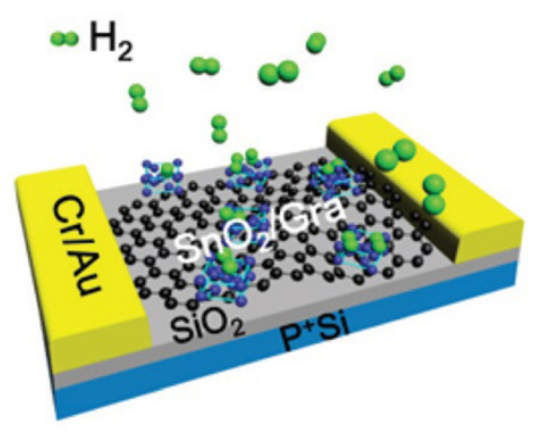

Figure 6. (a) The schematic of graphene transistor without obvious sensitivity to hydrogen; (b) The schematic of graphene field effect transistor (FET) decorated by metal oxide NPs with obvious sensitivity to hydrogen. Reprinted with permission from Ref. [131]. Copyright, 2015, Royal Society of Chemistry.

Recently, graphene/rGO functionalized with polymers has also emerged as new gas sensing materials due to the improved gas sensing behavior. The investigation of the synergistic behavior of conducting polymers (CPs) such as polyaniline (PANI), polypyrrole (PPy), polypyrene and graphene/rGO for molecular gas sensing resulted in outstanding performance in terms of both sensitivity and selectivity [136]. Graphene-PANI nanocomposites showed much higher sensitivity to the presence of hydrogen gas compared to that of pristine graphene sheets and PANI nanofibers [137]. Bai et al. [138] have fabricated a chemoresistor-type gas sensor using lyophilized GO/PPy composite hydrogel prepared through chemical polymerization of pyrrole in GO aqueous suspension for testing its $\mathrm{NH}_{3}$ sensing property. They found that the resistance of the GO/PPy composite aerogel increased by about $40 \%$ within $600 \mathrm{~s}$, compared with only $7 \%$ increase in the case of gas sensor based on electropolymerized PPy film to 800 ppm $\mathrm{NH}_{3}$ gas (as shown in Figure 7). The superior performance of the composite aerogel along with simple and low-cost fabrication process could be employed for developing highly sensitive and economically feasible gas sensors. In another similar work, the unique electrical properties of $\mathrm{rGO}$ and PPy were combined for $\mathrm{NH}_{3}$ gas sensing application in which the performance comparison of $\mathrm{rGO} / \mathrm{PPy}$ nanocomposite with intrinsic graphitic materials and nanocomposites of GO, graphene and graphite with PPy have been discussed [139]. The effective electron charge transfer between PPy and $\mathrm{NH}_{3}$ and the efficient transfer of resistance variation in PPy by the uniformly dispersed $\mathrm{rGO}$ in the $\mathrm{rGO} / \mathrm{PPy}$ nanocomposite enabled rapid and highly sensitive detection of ammonia gas, compared to PPy/GO, PPy/graphene, PPy/graphite and graphite. The excellent reproducibility of the nanocomposite based sensor was due to the ease of the recovery process at a lower temperature of $373 \mathrm{~K}$. 


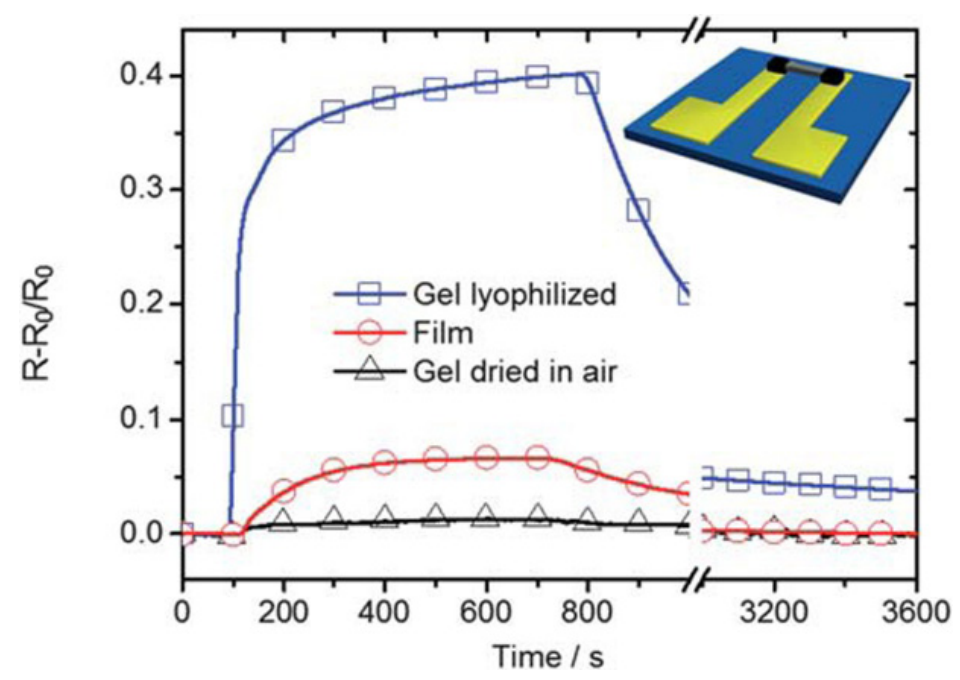

Figure 7. Ammonia gas sensing performance of three devices with sensing elements of lyophilized GO/PPy hydrogel, electrochemically deposited PPy film and GO/PPy hydrogel dried in air. Inset is a sketch of the gas sensor devices. Reprinted with permission from Ref. [138]. Copyright, 2011, Royal Society of Chemistry.

Recently, a chemoresistive gas sensor based on nanocomposites of graphene and polystyrene-sulfonate (rGO/PSS) was reported as a successful trimethylamine (TMA) gas detector. The rGO/PSS based gas sensor exhibited linear sensing response upon exposure to increasing TMA concentrations (23 to $183 \mathrm{mg} / \mathrm{L}$ ), along with good repeatability and reproducibility [140]. The decrease in resistance of the $\mathrm{GO} /(3,4$-ethylenedioxythiophene):poly(styrenesulfonate) (PEDOT:PSS) and rGO/PEDOT:PSS composite devices on hydrogen gas exposure suggested that nanocomposites of PEDOT:PSS with chemically modified graphene could be used effectively for hydrogen gas sensing, compared to pure PEDOT:PSS [141].

\subsection{Transition Metal Dichalcogenides (TMDs)}

Transition metal dichalcogenides are a group of materials with general formula of $\mathrm{MX}_{2}$, where $\mathrm{M}$ is a transition metal element of group IV, V or VI, and X is a chalcogen ( $\mathrm{S}$, Se or Te) [68]. Even though bulk crystals of TMDs were known and studied by researchers for decades, their 2D forms have received significant attention from both fundamental and application point of view after the success story of graphene [142]. These materials form two-dimensional layered structures in which the plane of metal atoms is sandwiched between two hexagonal planes of chalcogen atoms. Several 2D TMDs possess bandgap compared to pristine graphene [143,144] and hence these materials are promising candidates for new FETs with high on-off ratios and optoelectronic applications. The success of graphene based sensors greatly inspired scientists to explore the use of 2D TMDs as sensing materials. The electrical, optical and chemical properties of TMDs along with their high surface-to-volume ratio [143] suggest their applications in molecular sensing [68].

Molybdenum disulfide $\left(\mathrm{MoS}_{2}\right)$, one of the most popular semiconducting TMDs [144] has shown great promise for a variety of applications in electronics and optoelectronics [145-149]. As expected, gas sensors made from $\mathrm{MoS}_{2}$ have demonstrated excellent sensing characteristics such as high sensitivity, fast response time and good stability $[80-83,85]$. 
Several $\mathrm{MoS}_{2}$ based gas sensors using micromechanically exfoliated $[80,82,83]$ and liquid phase exfoliated [81] $\mathrm{MoS}_{2}$ flakes as sensing materials have been reported. Highly sensitive and stable detection of NO gas with detection limit as low as $0.8 \mathrm{ppm}$ had been shown by FET sensors using mechanically exfoliated multilayered (two- three- and four- layered) $\mathrm{MoS}_{2}$ sheets [80]. They observed strong chemisorption of $\mathrm{NO}$ on $\mathrm{n}$-doped $\mathrm{MoS}_{2}$ flakes, with a slow increase in resistivity of $\mathrm{MoS}_{2}$ flakes upon exposure to NO gas (over $30 \mathrm{~s}$ ), due to the induced p-type doping by the NO gas and also slow decrease in resistivity upon removal of NO. Flexible transistor sensor arrays based on $1.5 \mathrm{~mm}$-long $\mathrm{MoS}_{2}$ channel with rGO electrodes made on a polyethylene terephthalate (PET) substrate displayed much higher $\mathrm{NO}_{2}$ sensitivity and good reproducibility compared to novel rGO-FET sensors, which can be enhanced up to three times by functionalization of $\mathrm{MoS}_{2}$ with $\mathrm{Pt}$ nanoparticles [81]. But devices made from mechanically and liquid phase exfoliated $\mathrm{MoS}_{2}$ films suffer from poor scalability and poor electronic quality. $\mathrm{MoS}_{2}$ based devices made from more scalable approaches have also shown significant changes in electrical conductivity upon $\mathrm{NH}_{3}$ adsorption [85].

Recently, thin film transistors (TFTs) made from $\mathrm{MoS}_{2}$ demonstrated selective gas sensing behavior at operating temperature up to $220^{\circ} \mathrm{C}$, with much larger sensitivity [86] compared to similar graphene based gas sensors. TFT structures were fabricated by exfoliation from bulk $\mathrm{MoS}_{2}$ and were then transferred to $\mathrm{Si} / \mathrm{SiO}_{2}$ substrate with patterned contact electrodes. The working principle of these sensors are based on the generation of positive or negative charges at the $\mathrm{MoS}_{2}$ surface by the vapor molecules that either enhance or deplete the electron concentration in the channel depending on the type of the gas vapors. As the change in the channel conductance forms the primary basis for gas sensing, different gas species could not be distinguished from each other by looking at this conductance change. Selective gas sensing using graphene was already reported by considering two additional characteristics such as transient time and the peaks in the noise spectrum, when used together with the conductance change [51]. For different vapors such as acetone, acetonitrile, toluene and chloroform, the magnitude, sign of the response, and the response time constant are found to be different. The relative change in the device current and the characteristic transient times of the vapors were used as unique signatures [86]. On comparison, it was observed that for same vapor concentration, the relative resistance changes for graphene were less than $50 \%$. For acetonitrile, the increase in noise under the exposure is nearly an order of magnitude, whereas other gases showed a much smaller change.

Cantalini et al. [87] reported linear sensing response of layered $\mathrm{MoS}_{2}$ films to $\mathrm{NO}_{2}$ at concentrations below $1 \mathrm{ppm}$, with a detection limit of around $20 \mathrm{ppb}$ at $200{ }^{\circ} \mathrm{C}$. To $1 \mathrm{ppm} \mathrm{NO}$ gas in dry air at temperatures below $250{ }^{\circ} \mathrm{C}$, the chemically exfoliated $\mathrm{MoS}_{2}$ flakes showed typical p-type behavior with decreasing resistance. The $\mathrm{MoS}_{2}$ flakes showed reasonable sensitivity of 1.28 (ratio of resistance in air to resistance in $\mathrm{NO}_{2}$ ) to $1 \mathrm{ppm} \mathrm{NO} 2$ with fast and reversible response at $150{ }^{\circ} \mathrm{C}$. They did not show any response to $\mathrm{CO}$ and $\mathrm{H}_{2}$ at $150{ }^{\circ} \mathrm{C}$, enabling high selectivity. At temperatures above $250{ }^{\circ} \mathrm{C}$ in dry air, the $\mathrm{MoS}_{2}$ films showed p- to n-type transition of the electrical properties with response to 1 ppm $\mathrm{NO}_{2}$.

Planar sensor structures consisting of monolayer $\mathrm{MoS}_{2}$ channel on a $\mathrm{SiO}_{2} / \mathrm{Si}$ substrate with $\mathrm{Au}$ electrodes (as shown in Figure 8a,b) have shown rapid increase in $\mathrm{MoS}_{2}$ conductivity on exposure to triethylamine (TEA, a decomposition product of the V-series of nerve gas agent) and acetone. This sensor did not show any response to other analytes such as dichlorobenzene, dichloropentane, nitromethane, nitrotoluene and water vapor [83]. The observed initial response ( $\sim 5 \mathrm{~s})$ upon TEA 
exposure was more rapid than the $30 \mathrm{~s}$ reported previously for multilayer $\mathrm{MoS}_{2}$ to NO [80]. The response of the sensor channel to a sequence of 10 TEA pulses, each with a concentration of $0.002 \% P_{0}(\sim 1 \mathrm{ppm})$, was shown in Figure 9a. This monolayer $\mathrm{MoS}_{2}$ sensor (green curve in Figure 9a) exhibited no response to water vapor, used as a background constituent $\left(0.025 \% P_{0} \sim 6 \mathrm{ppm}\right)$, while a monolayer graphene sensor (purple curve in Figure 9a) showed a pronounced response to water vapor. For each TEA pulse, the change in conductivity followed similar pattern with an initial rapid rise and fall (as shown in Figure 9b). They observed increase in conductivity change with increase in the TEA concentration (as shown in Figure 9c) and the difference in relative sensitivity observed could be attributed to geometric factors and residual contamination during device fabrication. $\mathrm{MoS}_{2}$ based sensors showed a TEA detection threshold of $10 \mathrm{ppb}$ due to strong response and excellent signal-to-noise ratio. A comparison of the change in conductivity of these sensors with planar sensors fabricated from (a) monolayer graphene grown by chemical vapor deposition on copper and (b) a carbon nanotube (CNT) network consisting of a dense array of CNTs forming an electrically continuous thin film, as the sensor channel for a sequence of TEA pulses (10 s on, $20 \mathrm{~s}$ off) of $0.025 \%$ $P_{0}(12 \mathrm{ppm})$ concentration were plotted in Figure 9d. The response of the sensors based on CNTs and $\mathrm{MoS}_{2}$ to a single pulse of TEA are comparable, whereas those based on graphene, the response is observed to be small. Upon TEA exposure, the conductivity of both graphene and CNTs decrease, this is marked in contrast with response exhibited by $\mathrm{MoS}_{2}$. This could be attributed to the facts that TEA is a strong electron donor, CNT networks and graphene show p-type, whereas $\mathrm{MoS}_{2}$ show n-type character respectively. Hence the transient physisorption of TEA enhances the majority carrier density and the conductivity of $\mathrm{MoS}_{2}$, whereas these parameters decrease in the case of graphene and CNT network. The $\mathrm{MoS}_{2}$ sensors exhibited good sensitivity, high selectivity and a complementary response to CNT-network sensor [83].

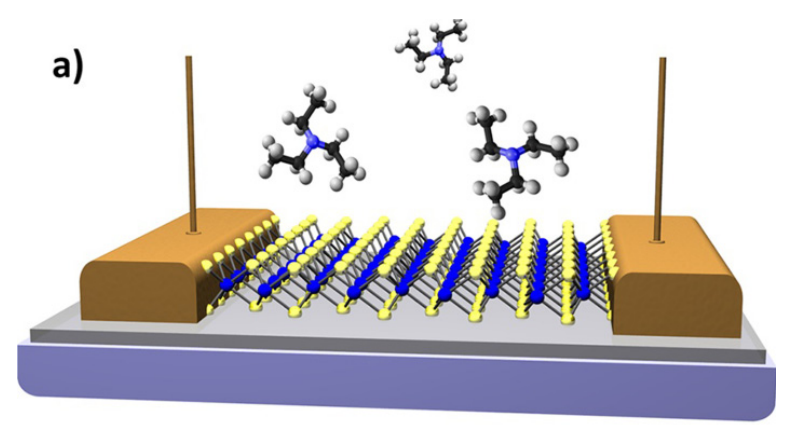

b)

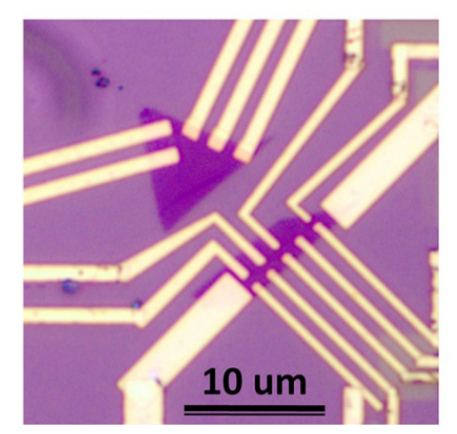

Figure 8. Schematic and image of the $\mathrm{MoS}_{2}$ monolayer sensor. (a) A single monolayer of $\mathrm{MoS}_{2}$ is supported on a $\mathrm{SiO}_{2} / \mathrm{Si}$ substrate and contacted with Au contact pads; (b) An optical image of the processed devices showing the monolayer $\mathrm{MoS}_{2}$ flakes electrically contacted by multiple Au leads. Reprinted with permission from Ref. [83]. Copyright, 2013, American Chemical Society. 

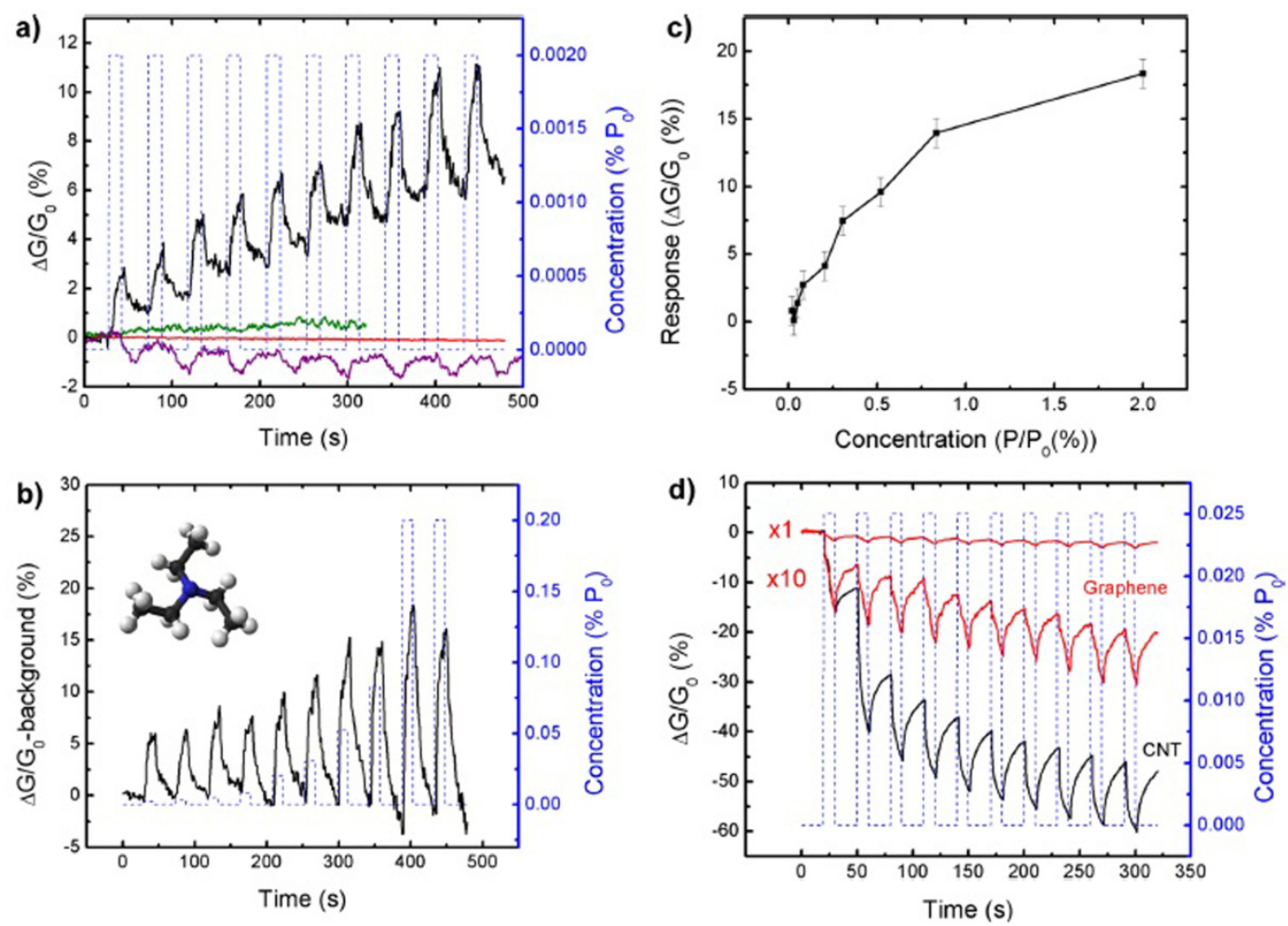

Figure 9. Response of sensors to triethylamine (TEA) exposure. (a) Change in conductivity of the monolayer $\mathrm{MoS}_{2}$ sensor channel upon exposure to a sequence of $0.002 \% P_{0}$ TEA pulses (black line). The dashed blue lines show the pulse timing $(15 \mathrm{~s}$ on/ $30 \mathrm{~s}$ off) and concentration. The solid red line shows the response to exposure of nitrogen only and serves as a control experiment. The solid green and purple lines show the response of the $\mathrm{MoS}_{2}$ and graphene sensors to water vapor pulses $\left(0.025 \% P_{0}\right)$, respectively; (b) Same as part a, but for a series of exposure pulses in which the TEA concentration increases from $0.002 \% P_{0}$ to $0.2 \% P_{0}$. A positive slope background has been removed. The inset shows a model of the TEA molecule, in which the nitrogen atom is blue, the carbon atoms are black, and the hydrogen atoms are light gray; (c) The amplitude of the conductivity change increases with TEA concentration. The vertical axis is the response to each individual pulse (not the time integrated response); (d) Change in conductivity of a CVD graphene monolayer (red) and CNT-network sensor (black) upon exposure to a sequence of $0.025 \% P_{0}$ TEA pulses (10 s on/20 s off). Reprinted with permission from Ref. [83]. Copyright, 2013, American Chemical Society.

Even though atomically thin films of TMDs synthesized by micromechanical cleavage, ultrasonication in organic solvents, aqueous surfactant solutions, or solutions of polymers in solvents, intercalation and exfoliation are well suited for applications in gas sensing [80-83], catalysis [150], composites [151], energy storage and conversion [152,153], the as prepared TMDs are not compatible with standard microlithography techniques in the nanodevice fabrication process. Gatensby and his co-workers [154] proposed a facile route for fabricating devices from $\mathrm{MoS}_{2}$ and $\mathrm{WS}_{2}$, grown by vapor phase sulfurization of pre-deposited metal layers which allows the production of highly homogenous TMD films over large areas with fine control of thickness from bulk to monolayer. Using shadow 
mask lithography, well-defined geometries were produced that could be easily integrated with standard micro-processing methods. The metal was deposited in selective areas by shadow masks, the sample was then sulfurized, interdigitated electrode (IDE) contacts were defined using a second shadow mask and were then sputtered. On exposure of the n-type sensing device to $\mathrm{NH}_{3}$ (electron donor), the conductivity increased due to the rise in majority carrier concentration. The fabricated sensor responded very quickly to ppb levels of $\mathrm{NH}_{3}$ with ultrahigh sensitivity down to $400 \mathrm{ppb}$. Similar to other nanomaterial-based sensors, these sensors also showed slow recovery in pure $\mathrm{N}_{2}$ flow at room temperature due to the strong binding with $\mathrm{NH}_{3}$ which thus required UV illumination or high temperature annealing for accelerating the recovery. The ease of device manufacture, cost effectiveness, scalability and compatibility with existing semiconductor fabrication methods make this process favorable for future sensors and other electronic applications [154].

Donarelli et al. [84] reported resistive type $\mathrm{MoS}_{2}$ based gas sensors which showed good response to $\mathrm{NO}_{2}, \mathrm{H}_{2}$ and relative humidity. The sensor device was fabricated by depositing liquid chemically exfoliated (in N-methyl pyrrolidone, NMP) $\mathrm{MoS}_{2}$ flakes on $\mathrm{Si}_{3} \mathrm{~N}_{4}$ substrate with pre-patterned IDEs on the front side, and on the back side with heater circuit for thermal annealing and $\mathrm{Pt}$ sensor for temperature control. The $\mathrm{MoS}_{2}$ based sensing device with $150{ }^{\circ} \mathrm{C}$ thermal annealing of exfoliated $\mathrm{MoS}_{2}$ flakes did not respond to $1 \mathrm{ppm} \mathrm{NO} 2$ at room temperature, but these $\mathrm{MoS}_{2}$ flakes responded at higher operating temperatures. The " $150{ }^{\circ} \mathrm{C}$ annealed" device, with p-type semiconducting behavior showed a decrease in resistance upon exposure to $\mathrm{NO}_{2}$ (oxidizing gas) in the $25-200{ }^{\circ} \mathrm{C}$ operating temperature range [84]. This " $150{ }^{\circ} \mathrm{C}$ annealed" device, exhibited good $\mathrm{NO}_{2}$ response either at $150{ }^{\circ} \mathrm{C}$ (ratio of resistance in air to that in $\mathrm{NO}_{2}$ is 1.29 ) or $200{ }^{\circ} \mathrm{C}$ (ratio of resistance in air to that in $\mathrm{NO}_{2}$ is 1.15). The " $250{ }^{\circ} \mathrm{C}$ annealed" device, with n-type semiconducting behavior showed an increase in resistance upon exposure to $\mathrm{NO}_{2}$, with significant resistance change at room temperature. The " $150{ }^{\circ} \mathrm{C}$ annealed" device was found to be faster than the " $250{ }^{\circ} \mathrm{C}$ annealed" device during adsorption. The response intensity of n-type $\mathrm{MoS}_{2}$ device exceeded by a factor of 5.0 (2.4) than that measured for the p-type device at $200{ }^{\circ} \mathrm{C}\left(250{ }^{\circ} \mathrm{C}\right) . " 250{ }^{\circ} \mathrm{C}$ annealed" device set at $200{ }^{\circ} \mathrm{C}$ showed outstanding performance with detection limit of $20 \mathrm{ppb}$ and response intensity equal to 5.80 at $1 \mathrm{ppm}$ concentration, compared to previously reported sensors. The n-MoS 2 has higher responses than GO based sensors, but with comparable responses to metal oxide and multi-walled CNT based sensors. The Raman and X-ray Photoelectron Spectroscopy (XPS) analyses proved that increase of sulfur vacancies after thermal annealing at $250{ }^{\circ} \mathrm{C}$ and the partial surface oxidation of the $\mathrm{MoS}_{2}$ upper layers in the form of $\mathrm{MoO}_{3}$ resulted in the n-type behavior and also contributed to the outstanding performance of the " $250{ }^{\circ} \mathrm{C}$ annealed" $\mathrm{MoS}_{2}$ sensor [84].

$\mathrm{MoS}_{2}$ sheets have proved to be exciting candidates for high performance gas sensing in which all the sensing experiments were performed in an inert atmosphere. Under practical environmental conditions that includes oxygen, the properties of $\mathrm{MoS}_{2}$ gets strongly affected by the adsorption of oxygen which leads to cross sensitivity effects and limits its practical applicability. Recently, room temperature sensing of $\mathrm{NO}_{2}$ was reported using $\mathrm{MoS}_{2} \mathrm{NS}$ decorated with $\mathrm{SnO}_{2}$ nanocrystals $\left(\mathrm{MoS}_{2} / \mathrm{SnO}_{2}\right)$ due to the improvement in the stability of the $\mathrm{MoS}_{2}$ sheets in practical environment by functionalization with $\mathrm{SnO}_{2}$ nanocrystals. High sensitivity, good selectivity and repeatability to $\mathrm{NO}_{2}$ in practical dry air were also exhibited by this hybrid sensor, as compared to other MoS2 sensors [155]. 
Sarkar et al. investigated the hydrogen gas sensing performance of a $\mathrm{MoS}_{2}$-based FET functionalized with Pd NPs [156]. The change in the work function of Pd NPs induced by the adsorption of $\mathrm{H}_{2}$ led to decrease in p-type doping which was measured by the change in the current of the Pd NP functionalized $\mathrm{MoS}_{2}$ FET. There was only a negligible change in current upon exposure of the $\mathrm{MoS}_{2}$ FET without NPs to $3 \mathrm{ppm} \mathrm{H}_{2}$ (as shown in Figure 10a), but upon the incorporation of Pd NPs, there was large increase in current level of the n-type $\mathrm{MoS}_{2}$ device (as shown in Figure 10b). For $3 \mathrm{ppm} \mathrm{H}_{2}$ gas at room temperature, the sensitivity (ratio of change in conductivity/ current to the initial conductivity/ current) of bulk $\mathrm{MoS}_{2}$ reported earlier was much less than 1 [157], whereas the sensitivity value increased to about 5 for $\mathrm{MoS}_{2}$ decorated with Pd NPs, which could be attributed to the use of few layer $\mathrm{MoS}_{2}$, around $8 \mathrm{~nm}$ thick and also due to the operation of FET in the subthreshold region [156].
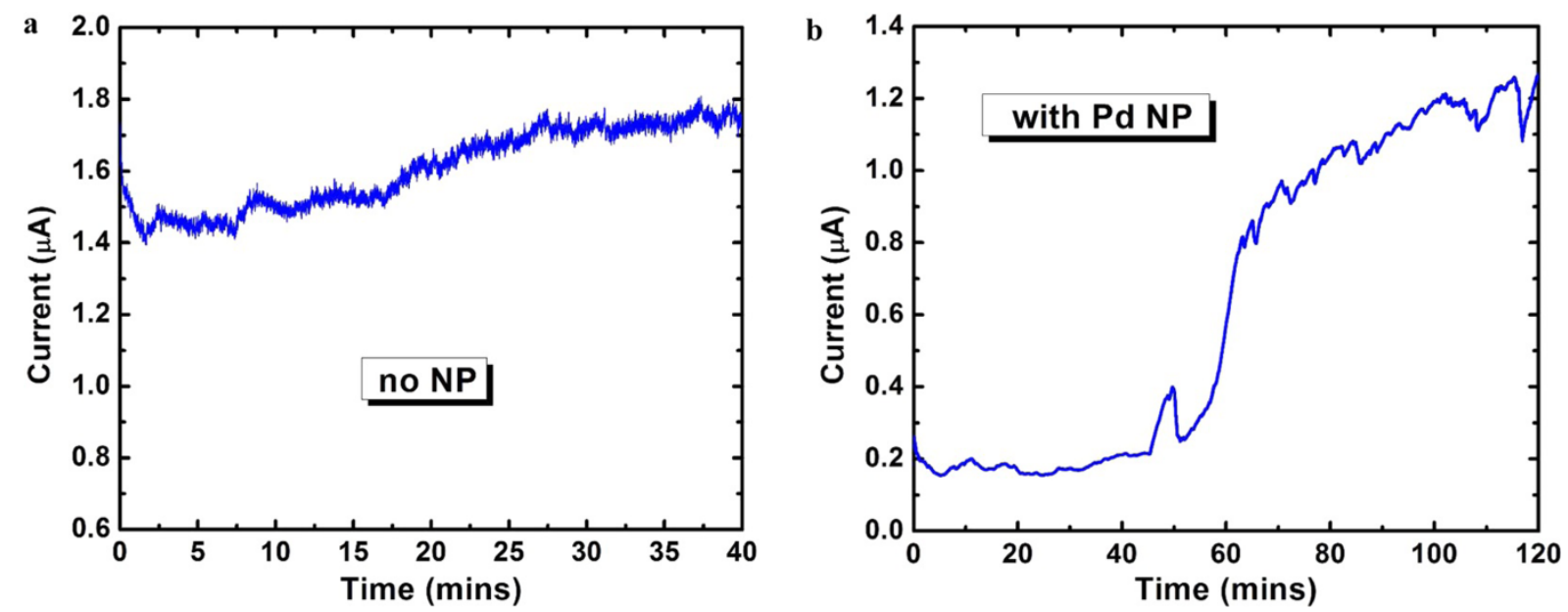

Figure 10. (a) Real-time measurement of current of $\mathrm{MoS}_{2}$ FET without any NPs. Thickness of $\mathrm{MoS}_{2}$ used was around $8 \mathrm{~nm}$; (b) Real-time measurement of current of the same $\mathrm{MoS}_{2}$ FET after incorporation of Pd NPs. Current increases substantially upon exposure to hydrogen ( $3 \mathrm{ppm}$ from time $=45$ min onwards) from $0.2 \mu \mathrm{A}$ to about $1 \mu \mathrm{A}$. Reprinted with permission from Ref. [156]. Copyright, 2015, American Chemical Society.

Even though many experimental reports on $\mathrm{MoS}_{2}$ based gas sensors exist, there have been very few reports on theoretical studies of the adsorption of gas molecules on $\mathrm{MoS}_{2}$ surface. In this direction, Yue et al. [158] analyzed the most stable adsorption position, orientation, associated charge transfer and the modification of electronic properties of the monolayer $\mathrm{MoS}_{2}$ surface due to the adsorption of $\mathrm{H}_{2}, \mathrm{O}_{2}, \mathrm{H}_{2} \mathrm{O}, \mathrm{NH}_{3}, \mathrm{NO}, \mathrm{NO}_{2}$, and $\mathrm{CO}$ using first-principles calculation based on DFT. They found that all these molecules acting as either electron donors or acceptors are only physisorbed on $\mathrm{MoS}_{2}$ surface, with small charge transfer and no significant alteration of band structure upon molecule adsorption. They also observed significant modulation of the charge transfer by the application of a perpendicular electric field [158]. Similarly, first principles simulation have also been employed by Zhao et al. [159] to investigate the adsorption of various gas molecules such as $\mathrm{CO}, \mathrm{CO}_{2}, \mathrm{NH}_{3}, \mathrm{NO}, \mathrm{NO}_{2}, \mathrm{CH}_{4}, \mathrm{H}_{2} \mathrm{O}, \mathrm{N}_{2}$, $\mathrm{O}_{2}$ and $\mathrm{SO}_{2}$ on monolayer $\mathrm{MoS}_{2}$ by including van der Waals interactions between the gas molecules and $\mathrm{MoS}_{2}$. They found that only $\mathrm{NO}, \mathrm{NO}_{2}$ and $\mathrm{SO}_{2}$ could bind strongly to $\mathrm{MoS}_{2}$ surface compared to other gas molecules, which was found to be in good agreement with experimental observations. 
Hence they suggested that $\mathrm{MoS}_{2}$ is more sensitive to $\mathrm{NO}, \mathrm{NO}_{2}$ and $\mathrm{SO}_{2}$ due to the observed charge transfer, variations in the electronic band structure and density of states (DOS) of $\mathrm{MoS}_{2}$ after gas adsorption [159].

Cho et al. developed a highly sensitive and selective gas sensor using uniform atomic-layered $\mathrm{MoS}_{2}$ synthesized by thermal CVD for the detection of $\mathrm{NO}_{2}$ and $\mathrm{NH}_{3}$ [160]. The charge transfer mechanism was evident from in situ photoluminescence characterizations and theoretical calculations. The first-principles DFT based calculations showed that adsorption processes are exothermic and thus probable adsorption of $\mathrm{NO}_{2}$ and $\mathrm{NH}_{3}$ onto $\mathrm{MoS}_{2}$ surface due to the negative adsorption energies for $\mathrm{NO}_{2}$ and $\mathrm{NH}_{3}$ gas molecules. The adsorption of $\mathrm{NO}_{2}$ onto $\mathrm{MoS}_{2}$ surface led to an increase in the intensity of the positively charged trion $\left(\mathrm{A}^{+}\right)$and suppression of the intensity of neutral exciton $\left(\mathrm{A}^{\circ}\right)$ due to the electron depletion of $\mathrm{MoS}_{2}$ by $\mathrm{NO}_{2}$ adsorption, whereas in the case of $\mathrm{NH}_{3}$ adsorption, the $\mathrm{A}^{\circ}$ peak intensity increased and the $\mathrm{A}^{+}$peak intensity decreased due to the electron accumulation of $\mathrm{MoS}_{2}$ by $\mathrm{NH}_{3}$ adsorption. The resistance of the $\mathrm{MoS}_{2}$ gas sensor exhibited an increase in the $\mathrm{NO}_{2}$ gas exposure mode, whereas the resistance exhibited a decrease during the $\mathrm{NH}_{3}$ gas exposure mode [160].

Recently, Cho et al. investigated the gas sensing performance of a 2D heterostructure-based gas sensor via the combination of mechanically exfoliated $\mathrm{MoS}_{2}$ and CVD grown graphene [161]. $\mathrm{MoS}_{2}$ flake based gas sensor with $\mathrm{Au} / \mathrm{Ti}$ metal electrodes exhibited excellent gas sensing stability over many sensing cycle tests with a detection limit of about 1.2 ppm to $\mathrm{NO}_{2}$. After $\mathrm{NO}_{2}$ injection, positive sensitivity i.e. increase in resistance was experienced by the $\mathrm{Au} / \mathrm{Ti} / \mathrm{MoS}_{2}$ based sensor, whereas in the case of $\mathrm{NH}_{3}$ injection, negative resistivity was observed. But the sensitivity of $\mathrm{MoS}_{2}$ based sensor to $\mathrm{NH}_{3}$ was found to be lower than that to $\mathrm{NO}_{2}$ due to the small charge transfer from $\mathrm{NH}_{3}$ and $\mathrm{MoS}_{2}$. The band structures of $\mathrm{MoS}_{2}$ after $\mathrm{NO}_{2}$ and $\mathrm{NH}_{3}$ adsorption also validated the high sensitivity of $\mathrm{MoS}_{2}$ to $\mathrm{NO}_{2}$. In the atomically thin heterostructure-based gas sensor, patterned graphene film was used instead of metal electrodes for the charge collection of $\mathrm{MoS}_{2}$. They gave a detailed explanation on the gas sensing mechanism of the graphene/ $\mathrm{MoS}_{2}$ device based on an equation having several variable resistance terms. 2D heterostructure-based structure fabricated on a flexible polyimide substrate retained its gas sensing characteristics without any serious performance degradation, even after harsh bending tests of about 5000 bending cycles. The flexible graphene/MoS 2 based sensor exhibited extraordinary long-term stability after 19 months. The graphene/MoS 2 based gas sensor experienced a decrease in resistance upon $\mathrm{NO}_{2}$ exposure and increase in resistance upon $\mathrm{NH}_{3}$ exposure, which is just the opposite trend of resistance change observed for $\mathrm{Au} / \mathrm{Ti} / \mathrm{MoS}_{2}$ based sensor due to the $\mathrm{n}$-type behavior of $\mathrm{Au} / \mathrm{Ti} / \mathrm{MoS}_{2}$ based device and $\mathrm{p}$-type behavior of graphene/MoS 2 heterojunction based gas sensor [161].

Cho et al. reported the bifunctional sensing characteristics of CVD synthesized $\mathrm{MoS}_{2}$ film to detect gas molecules and photons in a sequence [162]. The single $\mathrm{MoS}_{2}$ based device had demonstrated highly sensitive, selective detection of $\mathrm{NO}_{2}$ and also good photo sensing performance such as reasonable photoresponsivity, reliable photoresponse and rapid photoswitching. The observed sensing behavior of the $\mathrm{MoS}_{2}$ based device could be attributed to the charge transfer between $\mathrm{NO}_{2}$ and $\mathrm{MoS}_{2}$ sensing film and the detection limit was measured to be $120 \mathrm{ppb}$. The devices based on atomic-scale $\mathrm{MoS}_{2}$ films with bifunctional capability would pave the route towards the development of the futuristic multifunctional sensors [162]. 
Apart from $\mathrm{MoS}_{2}$, other members of TMDs such as $\mathrm{WS}_{2}$ and $\mathrm{MoSe}_{2}$ have also shown excellent gas sensing properties [88-91]. Researchers have explored the possibility of employing $\mathrm{WS}_{2}$ in gas sensing, as $\mathrm{WS}_{2}$ possess several advantages such as higher thermal stability, wider operation temperature range and favorable band structure as compared to $\mathrm{MoS}_{2}$. Huo et al. for the first time systematically studied the photoelectrical and gas sensing properties of transistors based on multilayer $\mathrm{WS}_{2}$ nanoflakes exfoliated from $\mathrm{WS}_{2}$ crystals [88]. The photoelectrical properties of multilayer $\mathrm{WS}_{2}$ nanoflake based FETs got strongly influenced by the gas molecules. Upon exposure to reducing gases such as ethanol and $\mathrm{NH}_{3}$, they observed strong and prolonged response with enhanced photo-responsivity and external quantum efficiency, due to the charge transfer between the physically adsorbed gas molecules and multilayer $\mathrm{WS}_{2}$ nanoflake based FETs [88]. High sensitivity detection of $\mathrm{WS}_{2}$ thin films towards $\mathrm{NH}_{3}$ was achieved by $\mathrm{WS}_{2}$ devices synthesized using inductively coupled plasma (ICP) source [89].

The interactions of $\mathrm{NH}_{3}$ and $\mathrm{H}_{2} \mathrm{O}$ molecules with monolayer $\mathrm{WS}_{2}$ investigated by means of first-principles calculations indicated that both $\mathrm{NH}_{3}$ and $\mathrm{H}_{2} \mathrm{O}$ molecules are physisorbed on monolayer $\mathrm{WS}_{2}$ [90]. The results from Bader charge analysis and plane-averaged differential charge density showed that $\mathrm{NH}_{3}$ and $\mathrm{H}_{2} \mathrm{O}$ act as electron donor and acceptor, leading to n- and p-type doping respectively. The charge transfer between the gas molecules and single-layer $\mathrm{WS}_{2}$ was primarily determined from the mixing of the highest occupied and lowest unoccupied molecular orbital with the underlying $\mathrm{WS}_{2}$ orbitals. They also provided detailed explanation about the sensing mechanism of the $\mathrm{WS}_{2}$-FET based gas sensor towards $\mathrm{NH}_{3}$ and $\mathrm{H}_{2} \mathrm{O}$. They found enhanced photoresponsivity and external quantum efficiency due to the increase in the total conduction electron density as more electrons are transferred from $\mathrm{NH}_{3}$ to the n-type $\mathrm{WS}_{2}$ channel, upon the adsorption of $\mathrm{NH}_{3}$ gas molecules. In the case of $\mathrm{H}_{2} \mathrm{O}$ adsorption on monolayer $\mathrm{WS}_{2}$, the source-drain current of $\mathrm{WS}_{2}$-FET based gas sensor got suppressed by the electron trapping of $\mathrm{H}_{2} \mathrm{O}$ molecule from the $\mathrm{WS}_{2}$ channel [90]. Their theoretical results were found to be in good agreement with the experimental results by Huo et al. [88]. After a single $\mathrm{NH}_{3}$ molecule adsorption on a $4 \times 4 \mathrm{WS}_{2}$ supercell, the saturation source-drain current ( $I_{\text {Dsat }}$ ) increased by $9.6 \times 10^{-6} \mathrm{~A}$ from its value under vacuum, which was found to be comparable to the experimental increment of dark drain current by $\sim 6.9 \times 10^{-7} \mathrm{~A}$ in $\mathrm{NH}_{3}$. Similarly $I_{\text {Dsat }}$ decreased by $2.8 \times 10^{-6} \mathrm{~A}$, consistent with the experimental decrement of dark drain current to $\sim 1.0 \times 10^{-8} \mathrm{~A}$ in air [90].

Late et al. developed a gas sensor based on single layer $\mathrm{MoSe}_{2}$ and the shift in the Raman spectra observed before and after exposing the $\mathrm{MoSe}_{2}$ based gas sensing device to $\mathrm{NH}_{3}$ confirmed the gas detection ability of single layer MoSe2. The detection limit of the MoSe2 based gas sensor was found to be $50 \mathrm{ppm}$. Their findings proved the potential of $\mathrm{MoSe}_{2}$ and other TMDs as excellent gas sensors [91].

\subsection{Phosphorene}

Motivated by the utility of 2D materials such as graphene and TMDs in nanodevice applications, scientists started searching for new 2D materials. Phosphorene, a single-atomic layer of black phosphorus (BP), arranged in a puckered honeycomb lattice [163] isolated recently, possess several advantageous properties such as existence of a finite band gap [164] and high charge carrier mobility 
of around $1000 \mathrm{~cm}^{2} / \mathrm{Vs}$ [165] over previously isolated 2D materials such as graphene and $\mathrm{MoS}_{2}$ with zero band gap and low mobility respectively. In addition to these, the anisotropic electrical conductance, high current on/off ratios, high operating frequencies, ambipolar behavior, fast and broadband photo detection of phosphorene [166,167] have already been exploited for FETs, PN junctions, photodetectors, solar cells, etc., [163,168-170] within the last year. Similar to graphene and $\mathrm{MoS}_{2}$, it was found that gas adsorption strongly influences the electrical properties of phosphorene, which could be effectively employed for gas sensing applications [92,93].

Kou et al. presented first-principles study of the adsorption of $\mathrm{CO}, \mathrm{CO}_{2}, \mathrm{NO}, \mathrm{NO}_{2}$ and $\mathrm{NH}_{3}$ on monolayer phosphorene. Their results proved superior gas sensing performance of phosphorene which even surpasses other 2D materials such as graphene and $\mathrm{MoS}_{2}$. Molecular doping of phosphorene by gas molecules due to the charge transfer between gas molecules and phosphorene resulted in large binding energies, similar to that observed in graphene and $\mathrm{MoS}_{2}$ [92]. The adsorption of gas molecules was observed to be much stronger than that in graphene and $\mathrm{MoS}_{2}$, which implies that monolayer phosphorene, can be used to make more sensitive gas sensors. The current-voltage (I-V) characteristics of phosphorene calculated using non-equilibrium Green's function (NEGF) formalism showed sensitive changes to adsorption, depending on the type of the gas molecule and thus leading to high selectivity.

The first experimental verification of gas sensors based on BP was reported by Abbas et al. recently [93]. FETs based on multilayer BP (as shown in Figure 11) showed increased conduction upon $\mathrm{NO}_{2}$ exposure (as shown in Figure 12a) due to the hole doping of the multilayer BP by $\mathrm{NO}_{2}$ and high sensitivity detection down to $5 \mathrm{ppb}$, which could be compared with the performance of other $2 \mathrm{D}$ materials. The relative conductance change for the multilayer BP based FET on exposure to varying $\mathrm{NO}_{2}$ concentrations of 5,10,20 and $40 \mathrm{ppb}$ followed the Langmuir isotherm for molecules adsorbed on a surface (as shown in Figure 12b). Moreover, the sensor had good stability even after repeated sensing cycles and better recovery after flushing with argon, which presented reversible adsorption and desorption process. Compared to other multilayer 2D materials such as $\mathrm{MoS}_{2}[81,82]$, the sensitivity of multilayer BP was found to be surprisingly very high [93]. The relative change in conductance of an $18 \mathrm{~nm}$ thick $\mathrm{MoS}_{2}$ flake to $1200 \mathrm{ppb} \mathrm{NO} 2$ was $\sim 1 \%$, whereas the conductance change for $55 \mathrm{~nm}$ thick BP to $5 \mathrm{ppb} \mathrm{NO}_{2}$ was $2.9 \%$. The high sensitivity of $\mathrm{BP}$ could be attributed to the high adsorption energies of $\mathrm{NO}_{2}$ on $\mathrm{BP}$ and the less out-of-plane conductance of BP compared to graphene and $\mathrm{MoS}_{2}$.

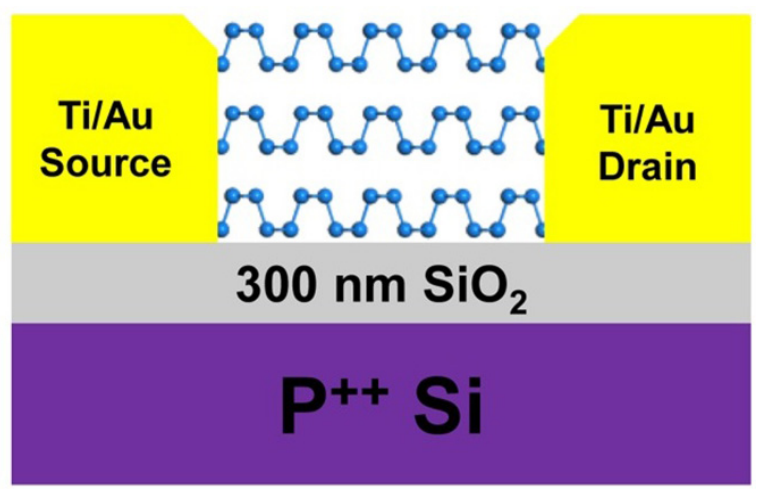

Figure 11. Scheme of a multilayer black phosphorus (BP) FET used for chemical sensing. Reprinted with permission from Ref. [93]. Copyright, 2015, American Chemical Society. 
a

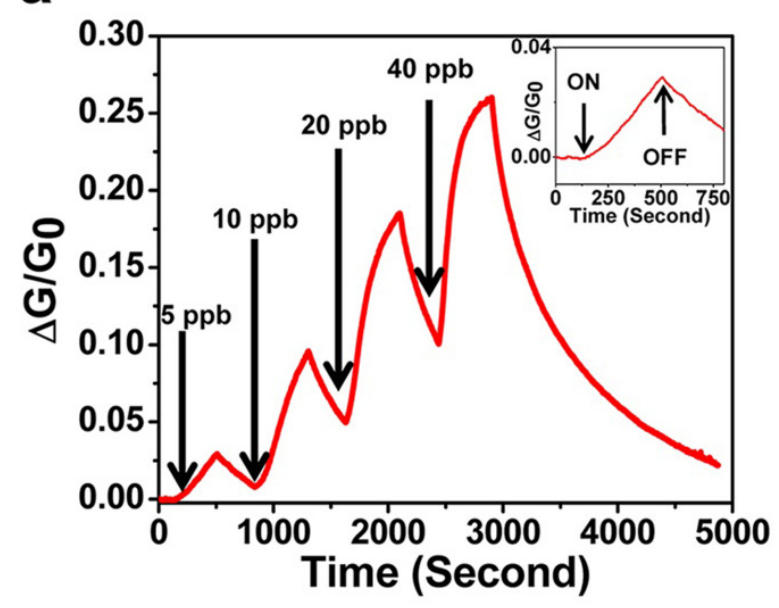

b

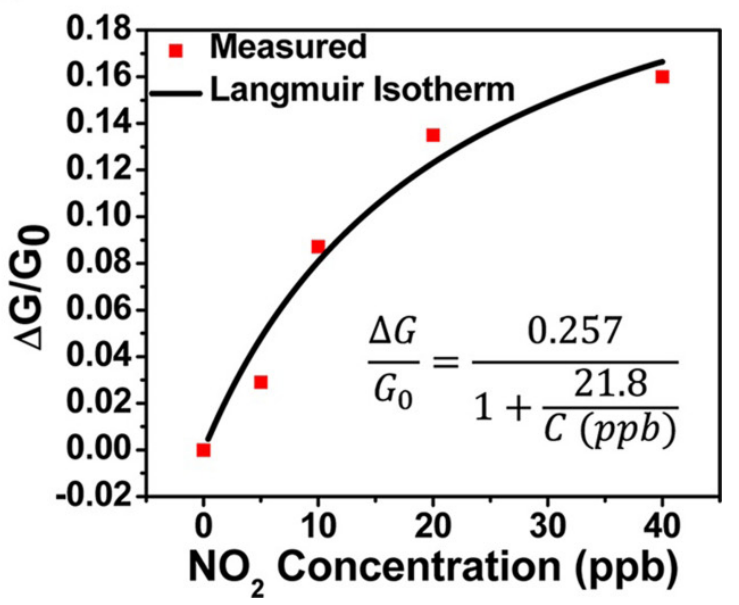

Figure 12. $\mathrm{NO}_{2}$ gas sensing performance of multilayer BP FET. (a) Relative conductance change $\left(\Delta \mathrm{G} / \mathrm{G}_{0}\right) v s$. time in seconds for a multilayer BP sensor. Inset shows a zoomed in image of a $5 \mathrm{ppb} \mathrm{NO} 2$ exposure response with identification of points in time where the $\mathrm{NO}_{2}$ gas is switched on and off; (b) $\Delta \mathrm{G} / \mathrm{G} 0$ plotted $v s$. $\mathrm{NO}_{2}$ concentration applied to the BP FET showing an agreement between the measured values (red squares) and the fitted Langmuir isotherm. Reprinted with permission from Ref. [93]. Copyright, 2015, American Chemical Society.

\subsection{D Semiconducting Metal Oxide Based Nanostructures}

Gas sensors based on metal oxide semiconductors such as $\mathrm{ZnO}, \mathrm{SnO}_{2}, \mathrm{WO}_{3}, \mathrm{Fe}_{2} \mathrm{O}_{3}, \mathrm{In}_{2} \mathrm{O}_{3}$, which are the most commonly used solid-state gas detection devices in domestic, industrial and commercial applications [171,172] operate on the principle of induced change in the electrical conductivity of the metal oxide due to the chemical reactions between gas molecules and semiconductor surface. But the sensitivity values reported for these sensors based on bulk materials or dense films were only moderate [172].

Compared to their bulk counterparts, 1D nanostructures in the form of nanowires, nanotubes, nanorods, nanoribbons, nanofibers, etc., provide large surface-to-volume ratios and high density surface reactive sites for gas sensing applications. The sensing platforms based on 1D nanostructured semiconducting metal oxides possess advantages of small size, light weight, good chemical and thermal stabilities under different operating conditions with minimal power consumption [4,173]. Gas sensors using 2D semiconducting metal oxide nanostructures such as nanosheets, nanowalls, nanoplates, nanoscale films have also attracted the major attention of researchers due to the their comparable or even better performance compared to 1D metal oxide nanostructures.

One of the most important and interesting metal oxide, $\mathrm{ZnO}$, a n-type semiconductor, has many unique optical and electrical properties such as wide band gap $(3.37 \mathrm{eV})$, high exciton binding energy $(60 \mathrm{meV})$ in addition to high temperature operation, high sensitivity to toxic and combustible gases, high thermal and chemical stability [174]. During the past, gas sensors based on $\mathrm{ZnO}$ were employed particularly for fuel leak detection in automobiles, space crafts, emissions from industrial processes, etc., Recently, $\mathrm{ZnO}$ nanostructures in the form of nanowires, nanotubes, nanorods, nanoflowers, etc., 
have shown superior performance in nanoscale optoelectronics [175,176], nanoscale piezotronics [177,178], catalysis and sensing devices [179-181].

Gas sensors made from 2D ZnO NSs with thickness of about 10-20 nm and width-to-thickness ratios of nearly one thousand, synthesized by simple mixed hydrothermal method in the presence of cetyltrimethyl ammonium bromide (CTAB) and 1,2-propanidiol, exhibited good selectivity and response to acetone and gasoline at high and low temperatures respectively in the presence of ammonia, ethanol and toluene [94]. For acetone, the ZnO NS based gas sensor showed increase in the response with increase in heating temperature, but for gasoline, the sensing behavior was found to be decreasing with rise in heating temperature. Both the response and recovery times got improved with increasing heating temperature. At $300{ }^{\circ} \mathrm{C}$, the response and recovery times were nearly $13 \mathrm{~s}$ and $30 \mathrm{~s}$ respectively. At $360^{\circ} \mathrm{C}$, the response to acetone (response magnitude of 31 ) was much higher than that of the other gases such as ammonia, ethanol, toluene and gasoline. At $180{ }^{\circ} \mathrm{C}$, the response to gasoline (response magnitude of 30) was also found to be much higher than that of the other gases. These results proved the potential of employing 2D ZnO NSs with extremely large specific surface area for selective detection of acetone and gasoline through temperature modulation [94].

$\mathrm{ZnO}$ nanowalls also have high surface-to-volume ratios and are highly effective in energy storage, field emission, chemical and biological sensing applications. Chang et al. [95] developed a CO gas sensor based on $\mathrm{ZnO}$ nanowalls grown on a glass substrate using fast, low-temperature, catalyst-free process in a tube furnace, compared to other $\mathrm{ZnO}$ nanowall fabrication methods which involve costly equipment, complex processes, toxic metal-organic precursors and flammable gases. They observed a linear trend of sensitivity ratio with the $\mathrm{CO}$ concentration. The current density-potential curve of the $\mathrm{ZnO}$ nanowall based gas sensor showed highest relative sensitivity ratio of 1.05 at $300{ }^{\circ} \mathrm{C}$ for 3000 ppm CO [95].

Earlier reports have shown enhancement in the gas sensing properties of the nanomaterials as their size approaches the Debye length, which is about $15 \mathrm{~nm}$ for $\mathrm{ZnO}$ at $325{ }^{\circ} \mathrm{C}$. Recently, ultrathin hexagonal $\mathrm{ZnO} \mathrm{NSs}$ as thin as $17 \mathrm{~nm}$ synthesized by simple hydrothermal method in the presence of CTAB exhibited the highest gas sensing response of 37.8 (ratio of resistance in air to that in target gas) at an operating temperature of $350^{\circ} \mathrm{C}$ with response and recovery times of $9 \mathrm{~s}$ and $11 \mathrm{~s}$ respectively to $50 \mathrm{ppm}$ formaldehyde gas [96].

As 2D porous metal oxide NSs with single-crystalline structure provide relatively large surface area due to the unique sheet-like morphology, they are successful gas sensing materials due to the enhanced sensing response and good stability. In the case of 2D NSs, the synthesis of single-crystalline structure with numerous pores was found to be easier as compared to 1D NWs [172]. Jing et al. [97] reported that gas sensor fabricated from porous $\mathrm{ZnO}$ nanoplates synthesized by simple microwave method showed strong response to chlorobenzene and ethanol at different operating temperatures. Liu et al. verified the gas sensing properties of novel single-crystalline $\mathrm{ZnO}$ NSs composed of porous interiors fabricated by annealing $\mathrm{ZnS}(\mathrm{en})_{0.5}$ (en = ethylenediamine) complex precursor [98]. The gas sensors fabricated from $\mathrm{ZnO}$ nanostructures exhibited high gas sensing response, short response time, fast recovery to formaldehyde and ammonia and also significant long term stability.

Hierarchically porous semiconducting metal oxide materials are proven to be ideal candidates for gas sensing applications due to the high surface area that they provide thanks to its peculiar structure which greatly facilitate significant enhancement in gas diffusion and mass transport, ultimately 
improving the sensitivity and response time of the gas sensor [182]. Due to ease of scale up, consistency and low power consumption, microstructure sensors based on thin films are appropriate for practical sensing applications. A microstructure sensor based on hierarchically porous $\mathrm{ZnO} \mathrm{NS}$ thin films demonstrated for the first time by Zeng et al. [99] showed the maximum response of 11.2 (ratio of resistance in dry air to that in target gas) at optimal temperature of $300{ }^{\circ} \mathrm{C}$ to $100 \mathrm{ppm} \mathrm{CO}$ with a response time of $25 \mathrm{~s}$, recovery time of $36 \mathrm{~s}$ and high selectivity to $\mathrm{CO}$ among other interfering gases such as $\mathrm{SO}_{2}, \mathrm{C}_{7} \mathrm{H}_{8}$. The comparison of the response of sensors based on $\mathrm{ZnO}$ NSs, $\mathrm{ZnO}$ NPs and $\mathrm{ZnO}$ seed layer as a function of $\mathrm{CO}$ concentration showed the fastest increase for ZnO NSs. Due to the hierarchically porous structure of ZnO NSs with well-defined and well-aligned micro-, meso- and nanoporosites, $\mathrm{ZnO}$ NS based sensors showed higher and fast response, selectivity with fast recovery compared to other $\mathrm{ZnO}$ based nanostructures [99].

Nanomaterials based on $\mathrm{NiO}$, a p-type semiconductor with excellent optical, electrical properties and high chemical stability have also proven as promising gas sensing materials owing to their high electron transport performance. Gas sensors based on $\mathrm{NiO}$ nanomaterials have demonstrated high sensitivity, low cost and good compatibility with micromachining. Zero-dimensional (0D) and 1D-NiO based sensors had excellent sensitivity, fast response and recovery, but suffer from poor stability due to poor interconnection between the sensing materials and electrodes on the sensing platform. $\mathrm{NiO}$ nanoscale films and foils, when used as sensing elements could overcome the stability issues associated with 0D- and 1D-NiO. Even though various approaches such as CVD, reactive sputtering, metal evaporation, sol-gel and chemical methods have been employed for preparing $2 \mathrm{D} \mathrm{NiO}$ nanostructures, $\mathrm{NiO}$ thin film prepared by chemical reduction has shown high gas sensing performance in terms of repeatability, sensitivity and stability due to high porosity, uniform morphology, continuity and nanocrystallinity of the films. Wang et al. [100] fabricated ammonia gas sensors based on $\mathrm{NiO}$ porous films on glass substrate through chemical reaction combined with high-temperature oxidation route in air, which showed fast response and excellent sensitivity (as shown in Figure 13). Without the need for pre-concentration step, the change in the sensor conductance reached up to about $18 \%$ upon exposure to $30 \mathrm{ppm} \mathrm{NH}_{3}$ for about $27 \mathrm{~s}$, which could be attributed to the large specific surface area and the porous surface structure of $2 \mathrm{D} \mathrm{NiO}$ film [100]. The high electron transport performance and good connection between the $2 \mathrm{D} \mathrm{NiO}$ films and the electrodes improved the recovery and stability of the sensor (as shown in Figure 13). They also observed excellent selectivity towards $\mathrm{NH}_{3}$ over other organic gases for the 2D NiO grainy film based sensor (as shown in Figure 14).

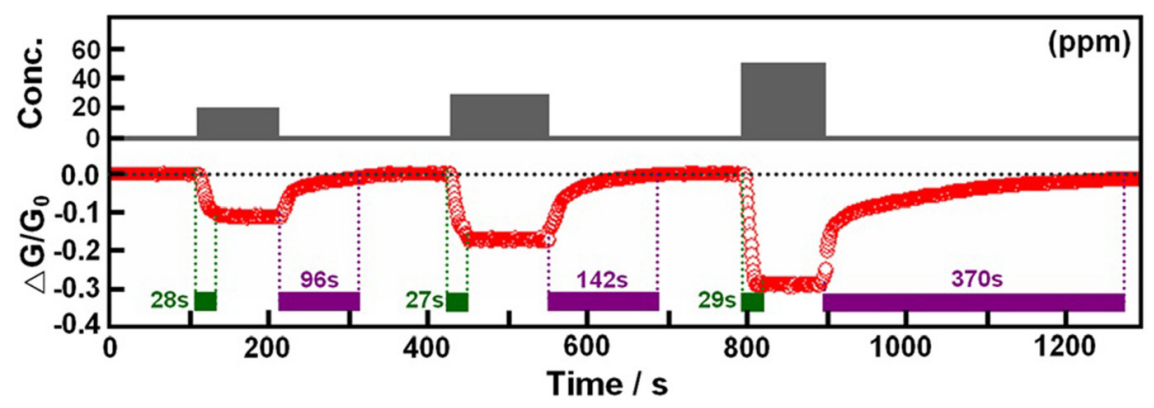

Figure 13. Stability and sensitivity test of gas sensors based on $\mathrm{NiO}$ films. The conductance change of $\mathrm{NiO}$ films on the glass substrates to 20,30 , and $50 \mathrm{ppm}$ of $\mathrm{NH}_{3}$ at room temperature [100]. 


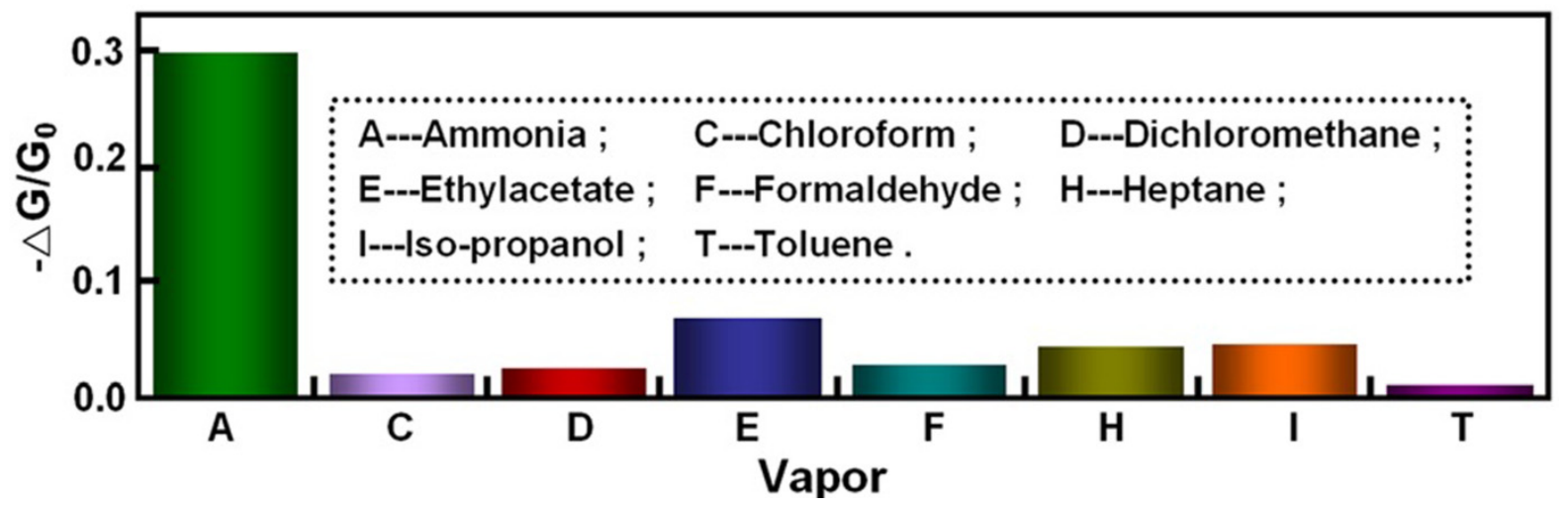

Figure 14. Selectivity tests of gas sensors based on $\mathrm{NiO}$ films. The concentration of $\mathrm{NH}_{3}$ is $50 \mathrm{ppm}$, and the concentration of other organic gases is higher than $300 \mathrm{ppm}$ [100].

$2 \mathrm{D}$ nanostructures made from $\mathrm{CuO}, \mathrm{WO}_{3}, \mathrm{SnO}_{2}$ have also shown good gas sensing characteristics. Uniform $\mathrm{CuO}$ nanosheets synthesized by mild hydrothermal method in the presence of CTAB showed stable and similar gas sensing response to combustible gases such as ethanol, acetone and gasoline [101]. 2D $\mathrm{WO}_{3}$ nanoplate based sensors showed superhigh sensitivity to different alcohols such as methanol, ethanol, isopropanol and butanol at operating temperatures of $260-360{ }^{\circ} \mathrm{C}$ [102]. The high specific surface area arising from the ultrathin plate like structure and the high crystallinity of $\mathrm{WO}_{3}$ nanoplates led to fast and effective adsorption of alcohol molecules. The response and recovery times for the $2 \mathrm{D}_{3} \mathrm{HO}_{3}$ nanoplate sensor were reported to be less than $15 \mathrm{~s}$ for all the tested alcohols.

A highly sensitive and fast responding $\mathrm{CO}$ sensor was demonstrated by $\mathrm{SnO}_{2} \mathrm{NSs}$ prepared by a facile chemical route at room temperature with subsequent high temperature annealing [103]. The reported response value of 2.34 and response time of $6 \mathrm{~s}$ were found to be significantly larger and less than those of $\mathrm{SnO}_{2}$ powders $(1.57,88 \mathrm{~s})$ respectively [103]. Sun et al. [106] have reported high response, good repeatability, short response and recovery periods, for $10 \mathrm{~nm}$ thick $\mathrm{SnO}_{2} \mathrm{NSs}$ prepared by simple and efficient hydrothermal method on exposure to ethanol. The response (ratio of resistance in air to that in tested gas atmosphere) of the $\mathrm{SnO}_{2} \mathrm{NS}$ sensor to $100 \mathrm{ppm}$ ethanol increased with the rise in the temperature from 200 to $250{ }^{\circ} \mathrm{C}$ which is followed by decrease in response with increase in operating temperature. The response had the maximum value of 73.3 at $250{ }^{\circ} \mathrm{C}$ for ethanol, much greater than the response values to other volatile organic compounds such as acetone, methanol, toluene, butanone and isopropanol. To $200 \mathrm{ppm} \mathrm{CO}$, the $\mathrm{SnO}_{2} \mathrm{NS}$ sensor showed the highest response at $300{ }^{\circ} \mathrm{C}$ with good repeatability and stability. Compared to other $\mathrm{SnO}_{2}$ nanostructures with longer response and recovery times, the response and recovery times of the $\mathrm{SnO}_{2} \mathrm{NS}$ based sensor were about 1 and $3 \mathrm{~s}$ respectively, believed to be due to the ease of gas diffusion towards the surface of $\mathrm{SnO}_{2} \mathrm{NSs}$ through the channels and pores and its reaction with the chemisorbed oxygen [106]. The CO gas sensing performance of different $\mathrm{SnO}_{2}$ NSs prepared through polyvinylpyrrolidone (PVP)-assisted hydrothermal method was investigated by Zeng et al. [108]. They found that the as prepared $\mathrm{SnO}_{2} \mathrm{NSs}$ showed a linear increase in sensing response with $\mathrm{CO}$ gas concentrations ranging from 100 to $450 \mathrm{ppm}$ at $300{ }^{\circ} \mathrm{C}$. Out of the different $\mathrm{SnO}_{2}$ NSs that vary in their PVP concentration, $\mathrm{SnO}_{2} \mathrm{NS}$ with the highest PVP concentration of $15 \mathrm{mM}$ exhibited good CO sensing performance such as higher response, fast response and recovery, due to the large contact surface area on the NSs [108]. 


\section{Conclusions and Future Perspectives}

The exfoliation of graphene and other 2D materials from their 3D counterparts and the recent utilization of their fascinating properties in various fields have been the main breakthrough in the realm of materials science. 2D materials possess great potential to play an important role in electronics, optoelectronics, energy conversion and storage, chemical and biological sensing due to their unique structural, electrical, physical and chemical properties. The success of new and improved nanodevices based on 2D materials such as $\mathrm{MoS}_{2}$, graphene and its derivatives in optoelectronics and nanoelectronics have inspired researchers to explore more $2 \mathrm{D}$ materials with better performance than those already isolated.

Gas sensors based on 2D materials have demonstrated high sensitivity detection of a wide variety of gas molecules at low concentrations, due to the maximum sensor surface area per unit volume, low noise and superior ability to screen charge fluctuations compared to $0 \mathrm{D}$ and $1 \mathrm{D}$ systems, in addition to their favorable electrical properties for gas adsorption. Even though pristine graphene, GO and rGO present a very promising gas sensing platform for room temperature gas detection, they get strongly affected by a range of different gas species and also suffer from slow recovery and poor electrical stability under different environmental conditions. Chemical modification of graphene or rGO, functionalized with defects, dopants, metal, metal oxide nanoparticles and polymers have proven to provide promising solution for improving the sensing performance with high specificity, enhanced sensitivity and low detection limit. Over the past two to three years, gas sensors based on other 2D materials such as TMDs and phosphorene have started gaining a lot of research interest due to their extraordinary and superior sensing capability over carbon based nanomaterials.

The desorption of gases on 2D materials such as $\mathrm{rGO}, \mathrm{MoS}_{2}$, phosphorene, etc., is observed to be slow due to the strong adsorption of gas molecules on these materials, which usually requires external assistance such as UV light exposure or high temperature annealing after gas exposure. This drawback of 2D material based gas sensors, which limits their practical use need to be addressed as it degrades the sensing performance in terms of sensitivity, detection limit and repeatability. Most of the gas sensing experiments already reported using 2D materials had to be performed under a controlled environment, as the sensitivity values were found to be affected by the presence of other gases in practical environmental conditions. Hence future work need to concentrate on achieving ultra-high selective gas sensing devices under practical conditions using 2D materials. This issue can be addressed by modification of the material surface with suitable modifiers such as metal or metal oxide nanoparticles. The functionalization scheme could be extended to TMDs and phosphorene, which had already proven to be highly successful for graphene based materials. Lack of large scale manufacturing of 2D materials with large area, high and uniform quality is yet another challenge. Flexible electronics could benefit from the mechanical compatibility of 2D materials with the device fabrication due to their excellent mechanical properties. 2D heterostructures obtained by the combination of different 2D materials provide a suitable sensing platform for developing future wearable electronics.

Modeling of nano sensing devices based on 2D materials is receiving great importance now-a-days, as it could enable the study of underlying sensing mechanisms and the analysis of the sensor performance before going for expensive experimentation. The electronic structure and quantum transport calculations of 2D material based electronic sensors before and after interaction with gas 
molecules are of great importance to the fabrication and development of novel nano sensors. Even though many reports on theoretical simulations of gas adsorption on graphene and modified graphene exist which give insights into the change in the electrical properties of graphene by gas molecule interactions, similar works on TMDs such as $\mathrm{MoS}_{2}, \mathrm{WS}_{2}, \mathrm{MoSe}_{2}$ and phosphorene are found to be very limited or lacking. Future progress in this field necessitates detailed understanding of the intrinsic properties of new 2D materials at the nano-scale and also the effect of adsorption of gas molecule on the electronic properties for evaluating the gas detection capability of these materials. First-principles calculations on the adsorption of gas molecules using different 2D materials could enable the identification of the suitable modification of these materials for enhanced sensor performance. The gas sensing mechanisms of devices based on newly discovered 2D materials are not completely explained till now. Once the above mentioned shortcomings are addressed, 2D materials could revolutionize the field of gas sensing so that they could emerge as ideal sensing elements with ultrahigh sensitivity, surprisingly high selectivity, fast response, good reversibility, room temperature operation, outstanding stability, etc., for gas sensing systems in the near future.

\section{Conflicts of Interest}

The authors declare no conflicts of interest.

\section{References}

1. Endres, H.E.; Göttler, W.; Hartinger, R.; Drost, S.; Hellmich, W.; Müller, G.; Braunmühl, C.B.-V.; Krenkow, A.; Perego, C.; Sberveglieri, G. A thin-film $\mathrm{SnO}_{2}$ sensor system for simultaneous detection of $\mathrm{CO}$ and $\mathrm{NO}_{2}$ with neural signal evaluation. Sens. Actuators B 1996, 36, 353-357.

2. Siyama, T.; Kato, A. A new detector for gaseous components using semiconductor thin film. Anal. Chem. 1962, 34, 1502-1503.

3. Tomchenko, A.A.; Harmer, G.P.; Marquis, B.T.; Allen, J.W. Semiconducting metal oxide sensor array for the selective detection of combustion gases. Sens. Actuators B 2003, 93, 126-134.

4. Arafat, M.; Dinan, B.; Akbar, S.A.; Haseeb, A. Gas sensors based on one dimensional nanostructured metal-oxides: A review. Sensors 2012, 12, 7207-7258.

5. Barsan, N.; Weimar, U. Conduction model of metal oxide gas sensors. J. Electroceramics 2001, 7, 143-167.

6. Capone, S.; Forleo, A.; Francioso, L.; Rella, R.; Siciliano, P.; Spadavecchia, J.; Presicce, D.; Taurino, A. Solid state gas sensors: State of the art and future activities. J. Optoelectron. Adv. Mater. 2003, 5, 1335-1348.

7. Jimenez-Cadena, G.; Riu, J.; Rius, F.X. Gas sensors based on nanostructured materials. Analyst 2007, 132, 1083-1099.

8. Hanna Varghese, S.; Nair, R.; G. Nair, Baiju; Hanajiri, T.; Maekawa, T.; Yoshida, Y.; Sakthi Kumar, D. Sensors based on carbon nanotubes and their applications: A review. Curr. Nanosci. 2010, 6, 331-346.

9. Kong, J.; Franklin, N.R.; Zhou, C.; Chapline, M.G.; Peng, S.; Cho, K.; Dai, H. Nanotube molecular wires as chemical sensors. Science 2000, 287, 622-625. 
10. Tabib-Azar, M.; Yan, X. Sensitive $\mathrm{NH}_{3} \mathrm{OH}$ and $\mathrm{HCl}$ gas sensors using self-aligned and self-welded multiwalled carbon nanotubes. IEEE Sens. J. 2007, 7, 1435-1439.

11. Li, J.; Lu, Y.; Ye, Q.; Cinke, M.; Han, J.; Meyyappan, M. Carbon nanotube sensors for gas and organic vapor detection. Nano Lett. 2003, 3, 929-933.

12. Varghese, O.K.; Kichambre, P.D.; Gong, D.; Ong, K.G.; Dickey, E.C.; Grimes, C.A. Gas sensing characteristics of multi-wall carbon nanotubes. Sens. Actuators B 2001, 81, 32-41.

13. Comini, E.; Sberveglieri, G. Metal oxide nanowires as chemical sensors. Mater. Today 2010, 13, $36-44$.

14. Cui, Y.; Wei, Q.; Park, H.; Lieber, C.M. Nanowire nanosensors for highly sensitive and selective detection of biological and chemical species. Science 2001, 293, 1289-1292.

15. Chen, X.; Wong, C.K.Y.; Yuan, C.A.; Zhang, G. Nanowire-based gas sensors. Sens. Actuators B 2013, 177, 178-195.

16. Liu, X.; Cheng, S.; Liu, H.; Hu, S.; Zhang, D.; Ning, H. A survey on gas sensing technology. Sensors 2012, 12, 9635-9665.

17. Dan, Y.; Evoy, S.; Johnson, A. Chemical gas sensors based on nanowires. In Nanowire Research Progress; Nova Science Publisher: Hauppauge, NY, USA, 2008; pp. 95-128.

18. Huang, X.-J.; Choi, Y.-K. Chemical sensors based on nanostructured materials. Sens. Actuators $B$ 2007, 122, 659-671.

19. Comini, E. Metal oxide nano-crystals for gas sensing. Anal. Chim. Acta 2006, 568, 28-40.

20. Novoselov, K.S.; Geim, A.K.; Morozov, S.V.; Jiang, D.; Zhang, Y.; Dubonos, S.V.; Grigorieva, I.V.; Firsov, A.A. Electric field effect in atomically thin carbon films. Science 2004, 306, 666-669.

21. Geim, A.K.; Novoselov, K.S. The rise of graphene. Nat. Mater. 2007, 6, 183-191.

22. Avouris, P. Graphene: Electronic and photonic properties and devices. Nano Lett. 2010, 10, 4285-4294.

23. Schwierz, F. Graphene transistors. Nat. Nano 2010, 5, 487-496.

24. Bonaccorso, F.; Sun, Z.; Hasan, T.; Ferrari, A.C. Graphene photonics and optoelectronics. Nat. Photonics 2010, 4, 611-622.

25. Pumera, M. Graphene-based nanomaterials for energy storage. Energy Environ. Sci. 2011, 4, 668-674.

26. Brownson, D.A.C.; Kampouris, D.K.; Banks, C.E. An overview of graphene in energy production and storage applications. J. Power Sources 2011, 196, 4873-4885.

27. Gwon, H.; Kim, H.-S.; Lee, K.U.; Seo, D.-H.; Park, Y.C.; Lee, Y.-S.; Ahn, B.T.; Kang, K. Flexible energy storage devices based on graphene paper. Energy Environ. Sci. 2011, 4, 1277-1283.

28. Huang, X.; Qi, X.; Boey, F.; Zhang, H. Graphene-based composites. Chem. Soc. Rev. 2012, 41, $666-686$.

29. Choi, H.-J.; Jung, S.-M.; Seo, J.-M.; Chang, D.W.; Dai, L.; Baek, J.-B. Graphene for energy conversion and storage in fuel cells and supercapacitors. Nano Energy 2012, 1, 534-551.

30. Wujcik, E.K.; Monty, C.N. Nanotechnology for implantable sensors: Carbon nanotubes and graphene in medicine. Wiley Interdiscip. Rev. 2013, 5, 233-249. 
31. Feng, L.; Liu, Z. Graphene in biomedicine: Opportunities and challenges. Nanomedicine 2011, 6 , 317-324.

32. Shen, H.; Zhang, L.; Liu, M.; Zhang, Z. Biomedical applications of graphene. Theranostics 2012 , 2, 283-294.

33. Chung, C.; Kim, Y.-K.; Shin, D.; Ryoo, S.-R.; Hong, B.H.; Min, D.-H. Biomedical applications of graphene and graphene oxide. Acc. Chem. Res. 2013, 46, 2211-2224.

34. Feng, L.; Wu, L.; Qu, X. New horizons for diagnostics and therapeutic applications of graphene and graphene oxide. Adv. Mater. 2013, 25, 168-186.

35. Ratinac, K.R.; Yang, W.; Ringer, S.P.; Braet, F. Toward ubiquitous environmental gas sensors-Capitalizing on the promise of graphene. Environ. Sci. Technol. 2010, 44, 1167-1176.

36. Yavari, F.; Koratkar, N. Graphene-based chemical sensors. J. Phys. Chem. Lett. 2012, 3, 1746-1753.

37. Zhou, M.; Zhai, Y.; Dong, S. Electrochemical sensing and biosensing platform based on chemically reduced graphene oxide. Anal. Chem. 2009, 81, 5603-5613.

38. Liu, Y.; Dong, X.; Chen, P. Biological and chemical sensors based on graphene materials. Chem. Soci. Rev. 2012, 41, 2283-2307.

39. Castro Neto, A.H.; Guinea, F.; Peres, N.M.R.; Novoselov, K.S.; Geim, A.K. The electronic properties of graphene. Rev. Mod. Phys. 2009, 81, 109-162.

40. Balandin, A.A. Thermal properties of graphene and nanostructured carbon materials. Nat. Mater. 2011, 10, 569-581.

41. Sandeep Kumar, V.; Venkatesh, A. Advances in graphene-based sensors and devices. J. Nanomed. Nanotechol. 2013, 4, e127.

42. Lee, C.; Wei, X.; Kysar, J.W.; Hone, J. Measurement of the elastic properties and intrinsic strength of monolayer graphene. Science 2008, 321, 385-388.

43. Wehling, T.O.; Novoselov, K.S.; Morozov, S.V.; Vdovin, E.E.; Katsnelson, M.I.; Geim, A.K.; Lichtenstein, A.I. Molecular doping of graphene. Nano Lett. 2008, 8, 173-177.

44. Schedin, F.; Geim, A.K.; Morozov, S.V.; Hill, E.W.; Blake, P.; Katsnelson, M.I.; Novoselov, K.S. Detection of individual gas molecules adsorbed on graphene. Nat. Mater. 2007, 6, 652-655.

45. Leenaerts, O.; Partoens, B.; Peeters, F.M. Adsorption of $\mathrm{H}_{2} \mathrm{O}, \mathrm{NH}_{3}, \mathrm{CO}, \mathrm{NO}_{2}$, and $\mathrm{NO}$ on graphene: A first-principles study. Phys. Rev. B 2008, 77, 125416.

46. Leenaerts, O.; Partoens, B.; Peeters, F.M. Adsorption of small molecules on graphene. Microelectron. J. 2009, 40, 860-862.

47. Ko, G.; Kim, H.Y.; Ahn, J.; Park, Y.M.; Lee, K.Y.; Kim, J. Graphene-based nitrogen dioxide gas sensors. Curr. Appl. Phys. 2010, 10, 1002-1004.

48. Yoon, H.J.; Jun, D.H.; Yang, J.H.; Zhou, Z.; Yang, S.S.; Cheng, M.M.-C. Carbon dioxide gas sensor using a graphene sheet. Sens. Actuators B 2011, 157, 310-313.

49. Romero, H.E.; Joshi, P.; Gupta, A.K.; Gutierrez, H.R.; Cole, M.W.; Tadigadapa, S.A.; Eklund, P.C. Adsorption of ammonia on graphene. Nanotechnology 2009, 20, 245501.

50. Chen, C.W.; Hung, S.C.; Yang, M.D.; Yeh, C.W.; Wu, C.H.; Chi, G.C.; Ren, F.; Pearton, S.J. Oxygen sensors made by monolayer graphene under room temperature. Appl. Phys. Lett. 2011, 99, 243502. 
51. Rumyantsev, S.; Liu, G.; Shur, M.S.; Potyrailo, R.A.; Balandin, A.A. Selective gas sensing with a single pristine graphene transistor. Nano Lett. 2012, 12, 2294-2298.

52. Chen, G.; Paronyan, T.M.; Harutyunyan, A.R. Sub-ppt gas detection with pristine graphene. Appl. Phys. Lett. 2012, 101, 053119.

53. Fattah, A.; Khatami, S. Selective $\mathrm{H}_{2} \mathrm{~S}$ gas sensing with a graphene/n-si schottky diode. IEEE Sens. J. 2014, 14, 4104-4108.

54. Nemade, K.R.; Waghuley, S.A. Chemiresistive gas sensing by few-layered graphene. J. Electron. Mater. 2013, 42, 2857-2866.

55. Kumar, S.; Kaushik, S.; Pratap, R.; Raghavan, S. Graphene on paper: A simple, low-cost chemical sensing platform. ACS Appl. Mater. Interfaces 2015, 7, 2189-2194.

56. Ricciardella, F.; Alfano, B.; Loffredo, F.; Villani, F.; Polichetti, T.; Miglietta, M.L.; Massera, E.; di Francia, G. Inkjet Printed Graphene-Based Chemi-Resistors for Gas Detection in Environmental Conditions. In Proceedings of the AISEM Annual Conference, 2015 XVIII, Trento, Italy, 3-5 February 2015; pp. 1-4.

57. Ganji, M.D.; Hosseini-Khah, S.; Amini-Tabar, Z. Theoretical insight into hydrogen adsorption onto graphene: A first-principles B3LYP-D3 study. Phys. Chem. Chem. Phys. 2015, 17, 2504-2511.

58. Prezioso, S.; Perrozzi, F.; Giancaterini, L.; Cantalini, C.; Treossi, E.; Palermo, V.; Nardone, M.; Santucci, S.; Ottaviano, L. Graphene oxide as a practical solution to high sensitivity gas sensing. J. Phys. Chem. C 2013, 117, 10683-10690.

59. Bi, H.; Yin, K.; Xie, X.; Ji, J.; Wan, S.; Sun, L.; Terrones, M.; Dresselhaus, M.S. Ultrahigh humidity sensitivity of graphene oxide. Sci. Rep. 2013, doi:10.1038/srep02714.

60. Peng, Y.; Li, J. Ammonia adsorption on graphene and graphene oxide: A first-principles study. Front. Environ. Sci. Eng. 2013, 7, 403-411.

61. Tang, S.; Cao, Z. Adsorption of nitrogen oxides on graphene and graphene oxides: Insights from density functional calculations. J. Chem. Phys. 2011, 134, 044710.

62. Robinson, J.T.; Perkins, F.K.; Snow, E.S.; Wei, Z.; Sheehan, P.E. Reduced graphene oxide molecular sensors. Nano Lett. 2008, 8, 3137-3140.

63. Lu, G.; Park, S.; Yu, K.; Ruoff, R.S.; Ocola, L.E.; Rosenmann, D.; Chen, J. Toward practical gas sensing with highly reduced graphene oxide: A new signal processing method to circumvent run-to-run and device-to-device variations. ACS Nano 2011, 5, 1154-1164.

64. Lipatov, A.; Varezhnikov, A.; Wilson, P.; Sysoev, V.; Kolmakov, A.; Sinitskii, A. Highly selective gas sensor arrays based on thermally reduced graphene oxide. Nanoscale 2013, 5, 5426-5434.

65. Hassinen, J.; Kauppila, J.; Leiro, J.; Määttänen, A.; Ihalainen, P.; Peltonen, J.; Lukkari, J. Low-cost reduced graphene oxide-based conductometric nitrogen dioxide-sensitive sensor on paper. Anal. Bioanal. Chem. 2013, 405, 3611-3617.

66. Wang, D.; Hu, Y.; Zhao, J.; Zeng, L.; Tao, X.; Chen, W. Holey reduced graphene oxide nanosheets for high performance room temperature gas sensing. J. Mater. Chem. A 2014, 2 , 17415-17420.

67. Miró, P.; Audiffred, M.; Heine, T. An atlas of two-dimensional materials. Chem. Soc. Rev. 2014, 43, 6537-6554. 
68. Wang, Q.H.; Kalantar-Zadeh, K.; Kis, A.; Coleman, J.N.; Strano, M.S. Electronics and optoelectronics of two-dimensional transition metal dichalcogenides. Nat. Nano 2012, 7, 699-712.

69. Butler, S.Z.; Hollen, S.M.; Cao, L.; Cui, Y.; Gupta, J.A.; Gutiérrez, H.R.; Heinz, T.F.; Hong, S.S.; Huang, J.; Ismach, A.F.; et al. Progress, challenges, and opportunities in two-dimensional materials beyond graphene. ACS Nano 2013, 7, 2898-2926.

70. Schwierz, F.; Pezoldt, J.; Granzner, R. Two-dimensional materials and their prospects in transistor electronics. Nanoscale 2015, 7, 8261-8283.

71. Fiori, G.; Bonaccorso, F.; Iannaccone, G.; Palacios, T.; Neumaier, D.; Seabaugh, A.; Banerjee, S.K.; Colombo, L. Electronics based on two-dimensional materials. Nat. Nano 2014, 9 , 768-779.

72. Das, S.; Robinson, J.A.; Dubey, M.; Terrones, H.; Terrones, M. Beyond graphene: Progress in novel two-dimensional materials and van der waals solids. Annu. Rev. Mater. Res. 2015, 45, $1-27$.

73. Yazyev, O.V.; Chen, Y.P. Polycrystalline graphene and other two-dimensional materials. Nat. Nano 2014, 9, 755-767.

74. Das, S.; Kim, M.; Lee, J.-W.; Choi, W. Synthesis, properties, and applications of 2-D materials: A comprehensive review. Crit. Rev. Solid State Mater. Sci. 2014, 39, 231-252.

75. Rao, C.N.R.; Gopalakrishnan, K.; Maitra, U. Comparative study of potential applications of graphene, $\mathrm{MoS}_{2}$, and other two-dimensional materials in energy devices, sensors, and related areas. ACS Appl. Mater. Interfaces 2015, 7, 7809-7832.

76. Varghese, S.S.; Lonkar, S.; Singh, K.K.; Swaminathan, S.; Abdala, A. Recent advances in graphene based gas sensors. Sens. Actuators B 2015, 218, 160-183.

77. Yuan, W.; Shi, G. Graphene-based gas sensors. J. Mater. Chem. A 2013, 1, 10078-10091.

78. Basu, S.; Bhattacharyya, P. Recent developments on graphene and graphene oxide based solid state gas sensors. Sens. Actuators B 2012, 173, 1-21.

79. Novoselov, K.S.; Jiang, D.; Schedin, F.; Booth, T.J.; Khotkevich, V.V.; Morozov, S.V.; Geim, A.K. Two-dimensional atomic crystals. Proc. Natl. Acad. Sci. USA 2005, 102, 10451-10453.

80. Li, H.; Yin, Z.; He, Q.; Li, H.; Huang, X.; Lu, G.; Fam, D.W.H.; Tok, A.I.Y.; Zhang, Q.; Zhang, H. Fabrication of single- and multilayer $\mathrm{MoS}_{2}$ film-based field-effect transistors for sensing no at room temperature. Small 2012, 8, 63-67.

81. He, Q.; Zeng, Z.; Yin, Z.; Li, H.; Wu, S.; Huang, X.; Zhang, H. Fabrication of flexible $\mathrm{MoS}_{2}$ thin-film transistor arrays for practical gas-sensing applications. Small 2012, 8, 2994-2999.

82. Late, D.J.; Huang, Y.-K.; Liu, B.; Acharya, J.; Shirodkar, S.N.; Luo, J.; Yan, A.; Charles, D.; Waghmare, U.V.; Dravid, V.P., et al. Sensing behavior of atomically thin-layered $\mathrm{MoS}_{2}$ transistors. ACS Nano 2013, 7, 4879-4891.

83. Perkins, F.K.; Friedman, A.L.; Cobas, E.; Campbell, P.M.; Jernigan, G.G.; Jonker, B.T. Chemical vapor sensing with monolayer MoS 2 . Nano Lett. 2013, 13, 668-673.

84. Donarelli, M.; Prezioso, S.; Perrozzi, F.; Bisti, F.; Nardone, M.; Giancaterini, L.; Cantalini, C.; Ottaviano, L. Response to $\mathrm{NO}_{2}$ and other gases of resistive chemically exfoliated $\mathrm{MoS}_{2}$-based gas sensors. Sens. Actuators B 2015, 207 Part A, 602-613. 
85. Lee, K.; Gatensby, R.; McEvoy, N.; Hallam, T.; Duesberg, G.S. High-performance sensors based on molybdenum disulfide thin films. Adv. Mater. 2013, 25, 6699-6702.

86. Shur, M.; Rumyantsev, S.; Jiang, C.; Samnakay, R.; Renteria, J.; Balandin, A.A. Selective Gas Sensing with $\mathrm{MoS}_{2}$ Thin Film Transistors. In Proceedings of the SENSORS, 2014 IEEE, Valencia, Spain, 2-5 November 2014; pp. 55-57.

87. Cantalini, C.; Giancaterini, L.; Donarelli, M.; Santucci, S.; Ottaviano, L. $\mathrm{NO}_{2}$ Response to Few-Layers $\mathrm{MoS}_{2}$. In Proceedings of the IMCS 2012-The 14th International Meeting on Chemical Sensors, Nuremberg, Germany, 20-23 May 2012; pp. 1656-1659.

88. Huo, N.; Yang, S.; Wei, Z.; Li, S.-S.; Xia, J.-B.; Li, J. Photoresponsive and gas sensing field-effect transistors based on multilayer $\mathrm{WS}_{2}$ nanoflakes. Sci. Rep. 2014, 4, 5209.

89. O’Brien, M.; Lee, K.; Morrish, R.; Berner, N.C.; McEvoy, N.; Wolden, C.A.; Duesberg, G.S. Plasma assisted synthesis of $\mathrm{WS}_{2}$ for gas sensing applications. Chem. Phys. Lett. 2014, 615, 6-10.

90. Zhou, C.J.; Yang, W.H.; Wu, Y.P.; Lin, W.; Zhu, H.L. Theoretical study of the interaction of electron donor and acceptor molecules with monolayer WS 2. J. Phys. D 2015, 48, 285303.

91. Late, D.J.; Doneux, T.; Bougouma, M. Single-layer $\mathrm{MoSe}_{2}$ based $\mathrm{NH}_{3}$ gas sensor. Appl. Phys. Lett. 2014, 105, 233103.

92. Kou, L.; Frauenheim, T.; Chen, C. Phosphorene as a superior gas sensor: Selective adsorption and distinct I-V response. J. Phys. Chem. Lett. 2014, 5, 2675-2681.

93. Abbas, A.N.; Liu, B.; Chen, L.; Ma, Y.; Cong, S.; Aroonyadet, N.; Köpf, M.; Nilges, T.; Zhou, C. Black phosphorus gas sensors. ACS Nano 2015, 9, 5618-5624.

94. Fan, H.; Jia, X. Selective detection of acetone and gasoline by temperature modulation in zinc oxide nanosheets sensors. Solid State Ionics 2011, 192, 688-692.

95. Chang, S.-P.; Wen, C.-H.; Chang, S.-J. Two-dimensional $\mathrm{ZnO}$ nanowalls for gas sensor and photoelectrochemical applications. Electron. Mater. Lett. 2014, 10, 693-697.

96. Guo, W.; Fu, M.; Zhai, C.; Wang, Z. Hydrothermal synthesis and gas-sensing properties of ultrathin hexagonal $\mathrm{ZnO}$ nanosheets. Ceram. Int. 2014, 40, 2295-2298.

97. Jing, Z.; Zhan, J. Fabrication and gas-sensing properties of porous $\mathrm{ZnO}$ nanoplates. Adv. Mater. 2008, 20, 4547-4551.

98. Liu, J.; Guo, Z.; Meng, F.; Luo, T.; Li, M.; Liu, J. Novel porous single-crystalline zno nanosheets fabricated by annealing $\mathrm{ZnS}(\mathrm{en}) 0.5$ (en = ethylenediamine) precursor. Application in a gas sensor for indoor air contaminant detection. Nanotechnology 2009, 20, 125501.

99. Zeng, Y.; Qiao, L.; Bing, Y.; Wen, M.; Zou, B.; Zheng, W.; Zhang, T.; Zou, G. Development of microstructure $\mathrm{CO}$ sensor based on hierarchically porous $\mathrm{ZnO}$ nanosheet thin films. Sens. Actuators B 2012, 173, 897-902.

100. Wang, J.; Yang, P.; Wei, X.; Zhou, Z. Preparation of NiO two-dimensional grainy films and their high-performance gas sensors for ammonia detection. Nanoscale Res. Lett. 2015, 10, 1-6.

101. Jia, X.; Fan, H.; Yang, W. Hydrothermal synthesis and primary gas sensing properties of CuO nanosheets. J. Dispers. Sci. Technol. 2010, 31, 866-869.

102. Chen, D.; Hou, X.; Wen, H.; Wang, Y.; Wang, H.; Li, X.; Zhang, R.; Lu, H.; Xu, H.; Guan, S. The enhanced alcohol-sensing response of ultrathin $\mathrm{WO}_{3}$ nanoplates. Nanotechnology 2010, $21,035501$. 
103. Moon, C.S.; Kim, H.-R.; Auchterlonie, G.; Drennan, J.; Lee, J.-H. Highly sensitive and fast responding $\mathrm{CO}$ sensor using $\mathrm{SnO}_{2}$ nanosheets. Sens. Actuators B 2008, 131, 556-564.

104. Li, K.-M.; Li, Y.-J.; Lu, M.-Y.; Kuo, C.-I.; Chen, L.-J. Direct conversion of single-layer sno nanoplates to multi-layer $\mathrm{SnO}_{2}$ nanoplates with enhanced ethanol sensing properties. Adv. Funct. Mater. 2009, 19, 2453-2456.

105. Xu, M.-H.; Cai, F.-S.; Yin, J.; Yuan, Z.-H.; Bie, L.-J. Facile synthesis of highly ethanol-sensitive $\mathrm{SnO}_{2}$ nanosheets using homogeneous precipitation method. Sens. Actuators B 2010, 145, $875-878$.

106. Sun, P.; Cao, Y.; Liu, J.; Sun, Y.; Ma, J.; Lu, G. Dispersive $\mathrm{SnO}_{2}$ nanosheets: Hydrothermal synthesis and gas-sensing properties. Sens. Actuators B 2011, 156, 779-783.

107. Lou, Z.; Wang, L.; Wang, R.; Fei, T.; Zhang, T. Synthesis and ethanol sensing properties of $\mathrm{SnO}_{2}$ nanosheets via a simple hydrothermal route. Solid-State Electron. 2012, 76, 91-94.

108. Zeng, W.; Wu, M.; Li, Y.; Wu, S. Hydrothermal synthesis of different $\mathrm{SnO}_{2}$ nanosheets with co gas sensing properties. J. Mater. Sci.: Mater. Electron. 2013, 24, 3701-3706.

109. Du, X.; Skachko, I.; Barker, A.; Andrei, E.Y. Approaching ballistic transport in suspended graphene. Nat Nano 2008, 3, 491-495.

110. de Heer, W.A.; Berger, C.; Wu, X.; First, P.N.; Conrad, E.H.; Li, X.; Li, T.; Sprinkle, M.; Hass, J.; Sadowski, M.L.; et al. Epitaxial graphene. Solid State Commun. 2007, 143, 92-100.

111. Park, S.; Ruoff, R.S. Chemical methods for the production of graphenes. Nat Nano 2009, 4, 217-224.

112. Balandin, A.A.; Ghosh, S.; Bao, W.; Calizo, I.; Teweldebrhan, D.; Miao, F.; Lau, C.N. Superior thermal conductivity of single-layer graphene. Nano Lett. 2008, 8, 902-907.

113. Blake, P.; Hill, E.W.; Castro Neto, A.H.; Novoselov, K.S.; Jiang, D.; Yang, R.; Booth, T.J.; Geim, A.K. Making graphene visible. Appl. Phys. Lett. 2007, 91, 063124.

114. Dutta, P.; Horn, P.M. Low-frequency fluctuations in solids: 1/f Noise. Rev. Mod. Phys. 1981, 53, 497-516.

115. McAllister, M.J.; Li, J.-L.; Adamson, D.H.; Schniepp, H.C.; Abdala, A.A.; Liu, J.; Herrera-Alonso, M.; Milius, D.L.; Car, R.; Prud'homme, R.K.; et al. Single sheet functionalized graphene by oxidation and thermal expansion of graphite. Chemistry of Materials 2007, 19, 4396-4404.

116. Zhang, C.; Lv, W.; Xie, X.; Tang, D.; Liu, C.; Yang, Q.-H. Towards low temperature thermal exfoliation of graphite oxide for graphene production. Carbon 2013, 62, 11-24.

117. Stankovich, S.; Dikin, D.A.; Piner, R.D.; Kohlhaas, K.A.; Kleinhammes, A.; Jia, Y.; Wu, Y.; Nguyen, S.T.; Ruoff, R.S. Synthesis of graphene-based nanosheets via chemical reduction of exfoliated graphite oxide. Carbon 2007, 45, 1558-1565.

118. Si, Y.; Samulski, E.T. Synthesis of water soluble graphene. Nano Letters 2008, 8, 1679-1682.

119. Zhang, Y.-H.; Chen, Y.-B.; Zhou, K.-G.; Liu, C.-H.; Zeng, J.; Zhang, H.-L.; Peng, Y. Improving gas sensing properties of graphene by introducing dopants and defects: A first-principles study. Nanotechnology 2009, 20, 185504.

120. Shao, L.; Chen, G.; Ye, H.; Wu, Y.; Qiao, Z.; Zhu, Y.; Niu, H. Sulfur dioxide adsorbed on graphene and heteroatom-doped graphene: A first-principles study. Eur. Phys. J. B 2013, 86, 1-5. 
121. Zhang, H.; Luo, X.; Song, H.; Lin, X.; Lu, X.; Tang, Y. DFT study of adsorption and dissociation behavior of $\mathrm{H}_{2} \mathrm{~S}$ on Fe-doped graphene. Appl. Surface Sci. 2014, 317, 511-516.

122. Ma, C.; Shao, X.; Cao, D. Nitrogen-doped graphene as an excellent candidate for selective gas sensing. Sci. China Chem. 2014, 57, 911-917.

123. Liu, X.-Y.; Zhang, J.-M.; Xu, K.-W.; Ji, V. Improving $\mathrm{SO}_{2}$ gas sensing properties of graphene by introducing dopant and defect: A first-principles study. Appl. Surface Sci. 2014, 313, 405-410.

124. Zhou, Q.; Yuan, L.; Yang, X.; Fu, Z.; Tang, Y.; Wang, C.; Zhang, H. DFT study of formaldehyde adsorption on vacancy defected graphene doped with $\mathrm{B}, \mathrm{N}$, and S. Chem. Phys. 2014, 440, 80-86.

125. Wang, X.; Sun, G.; Routh, P.; Kim, D.-H.; Huang, W.; Chen, P. Heteroatom-doped graphene materials: Syntheses, properties and applications. Chem. Soc. Rev. 2014, 43, 7067-7098.

126. Lv, R.; Li, Q.; Botello-Méndez, A.R.; Hayashi, T.; Wang, B.; Berkdemir, A.; Hao, Q.; Elías, A.L.; Cruz-Silva, R.; Gutiérrez, H.R.; et al. Nitrogen-doped graphene: Beyond single substitution and enhanced molecular sensing. Sci. Rep. 2012, 2, 586.

127. Niu, F.; Liu, J.-M.; Tao, L.-M.; Wang, W.; Song, W.-G. Nitrogen and silica co-doped graphene nanosheets for $\mathrm{NO}_{2}$ gas sensing. J. Mater. Chem. A 2013, 1, 6130-6133.

128. Chung, M.G.; Kim, D.-H.; Seo, D.K.; Kim, T.; Im, H.U.; Lee, H.M.; Yoo, J.-B.; Hong, S.-H.; Kang, T.J.; Kim, Y.H. Flexible hydrogen sensors using graphene with palladium nanoparticle decoration. Sens. Actuators B 2012, 169, 387-392.

129. Cho, B.; Yoon, J.; Hahm, M.G.; Kim, D.-H.; Kim, A.R.; Kahng, Y.H.; Park, S.-W.; Lee, Y.-J.; Park, S.-G.; Kwon, J.-D. Graphene-based gas sensor: Metal decoration effect and application to a flexible device. J. Mater. Chem. C 2014, 2, 5280-5285.

130. Wang, J.; Rathi, S.; Singh, B.; Lee, I.; Maeng, S.; Joh, H.-I.; Kim, G.-H. Dielectrophoretic assembly of Pt nanoparticle-reduced graphene oxide nanohybrid for highly-sensitive multiple gas sensor. Sens. Actuators B 2015, 220, 755-761.

131. Zhang, Z.; Zou, X.; Xu, L.; Liao, L.; Liu, W.; Ho, J.; Xiao, X.; Jiang, C.; Li, J. Hydrogen gas sensor based on metal oxide nanoparticles decorated graphene transistor. Nanoscale 2015, 7, 10078-10084.

132. Liu, S.; Yu, B.; Zhang, H.; Fei, T.; Zhang, T. Enhancing $\mathrm{NO}_{2}$ gas sensing performances at room temperature based on reduced graphene oxide- $\mathrm{ZnO}$ nanoparticles hybrids. Sens. Actuators $B$ 2014, 202, 272-278.

133. Zhou, L.; Shen, F.; Tian, X.; Wang, D.; Zhang, T.; Chen, W. Stable $\mathrm{Cu}_{2} \mathrm{O}$ nanocrystals grown on functionalized graphene sheets and room temperature $\mathrm{H}_{2} \mathrm{~S}$ gas sensing with ultrahigh sensitivity. Nanoscale 2013, 5, 1564-1569.

134. Su, P.-G.; Peng, S.-L. Fabrication and $\mathrm{NO}_{2}$ gas-sensing properties of reduced graphene oxide/ $\mathrm{WO}_{3}$ nanocomposite films. Talanta 2015, 132, 398-405.

135. Jiang, Z.; Li, J.; Aslan, H.; Li, Q.; Li, Y.; Chen, M.; Huang, Y.; Froning, J.P.; Otyepka, M.; Zbořil, R. A high efficiency $\mathrm{H}_{2} \mathrm{~S}$ gas sensor material: Paper like $\mathrm{Fe}_{2} \mathrm{O}_{3}$ /graphene nanosheets and structural alignment dependency of device efficiency. J. Mater. Chem. A 2014, 2, 6714-6717.

136. Lei, W.; Si, W.; Xu, Y.; Gu, Z.; Hao, Q. Conducting polymer composites with graphene for use in chemical sensors and biosensors. Microchim. Acta 2014, 181, 707-722. 
137. Al-Mashat, L.; Shin, K.; Kalantar-zadeh, K.; Plessis, J.D.; Han, S.H.; Kojima, R.W.; Kaner, R.B.; Li, D.; Gou, X.; Ippolito, S.J.; et al. Graphene/polyaniline nanocomposite for hydrogen sensing. J. Phys. Chem. C 2010, 114, 16168-16173.

138. Bai, H.; Sheng, K.; Zhang, P.; Li, C.; Shi, G. Graphene oxide/conducting polymer composite hydrogels. J. Mater. Chem. 2011, 21, 18653-18658.

139. Jang, W.-K.; Yun, J.; Kim, H.-I.; Lee, Y.-S. Improvement of ammonia sensing properties of polypyrrole by nanocomposite with graphitic materials. Colloid Polym. Sci. 2013, 291, 1095-1103.

140. Ranola, R.A.G.; Concina, I.; Sevilla, F.B.; Ferroni, M.; Sangaletti, L.; Sberveglieri, G.; Comini, E. In Room Temperature Trimethylamine Gas Sensor Based on Aqueous Dispersed Graphene, Proceedings of the 2015 XVIII AISEM Annual Conference, Trento, Italy, 3-5 February 2015; pp. 1-4.

141. Zheng, Y.; Lee, D.; Koo, H.Y.; Maeng, S. Chemically modified graphene/PEDOT:PSS nanocomposite films for hydrogen gas sensing. Carbon 2015, 81, 54-62.

142. Ayari, A.; Cobas, E.; Ogundadegbe, O.; Fuhrer, M.S. Realization and electrical characterization of ultrathin crystals of layered transition-metal dichalcogenides. J. Appl. Phys. 2007, 101, 14507-14507.

143. Wilson, J.A.; Yoffe, A.D. The transition metal dichalcogenides discussion and interpretation of the observed optical, electrical and structural properties. Adv. Phys. 1969, 18, 193-335.

144. Mak, K.F.; Lee, C.; Hone, J.; Shan, J.; Heinz, T.F. Atomically thin $\mathrm{MoS}_{2}$ : A new direct-gap semiconductor. Phys. Rev. Lett. 2010, 105, 136805.

145. Izyumskaya, N.; Demchenko, D.O.; Avrutin, V.; Özgur, U.; Morkoc, H. Two-dimensional MoS 2 as a new material for electronic devices. Turkish J. Phys. 2014, 38, 478-496.

146. Li, X.; Zhu, H. Two-dimensional $\mathrm{MoS}_{2}$ : Properties, preparation, and applications. J. Materiomics 2015, 1, 33-44.

147. Radisavljevic, B; Radenovic, A; Brivio, J; Giacometti, V; Kis, A. Single-layer $\mathrm{MoS}_{2}$ transistors. Nat. Nano 2011, 6, 147-150.

148. Pu, J.; Yomogida, Y.; Liu, K.-K.; Li, L.-J.; Iwasa, Y.; Takenobu, T. Highly flexible $\mathrm{MoS}_{2}$ thin-film transistors with ion gel dielectrics. Nano Lett. 2012, 12, 4013-4017.

149. Lopez-Sanchez, O.; Lembke, D.; Kayci, M.; Radenovic, A.; Kis, A. Ultrasensitive photodetectors based on monolayer MoS2. Nat. Nano 2013, 8, 497-501.

150. Lukowski, M.A.; Daniel, A.S.; Meng, F.; Forticaux, A.; Li, L.; Jin, S. Enhanced hydrogen evolution catalysis from chemically exfoliated metallic $\mathrm{MoS}_{2}$ nanosheets. J. Am. Chem. Soc. 2013, 135, 10274-10277.

151. Tan, C.; Zhang, H. Two-dimensional transition metal dichalcogenide nanosheet-based composites. Chem. Soc. Rev. 2015, 44, 2713-2731.

152. Cao, X.; Shi, Y.; Shi, W.; Rui, X.; Yan, Q.; Kong, J.; Zhang, H. Preparation of MoS2-coated three-dimensional graphene networks for high-performance anode material in lithium-ion batteries. Small 2013, 9, 3433-3438.

153. Cao, L.; Yang, S.; Gao, W.; Liu, Z.; Gong, Y.; Ma, L.; Shi, G.; Lei, S.; Zhang, Y.; Zhang, S.; et al. Direct laser-patterned micro-supercapacitors from paintable $\mathrm{MoS}_{2}$ films. Small 2013, 9 , 2905-2910. 
154. Gatensby, R.; McEvoy, N.; Lee, K.; Hallam, T.; Berner, N.C.; Rezvani, E.; Winters, S.; O'Brien, M.; Duesberg, G.S. Controlled synthesis of transition metal dichalcogenide thin films for electronic applications. Appl. Surface Sci. 2014, 297, 139-146.

155. Cui, S.; Wen, Z.; Huang, X.; Chang, J.; Chen, J. Stabilizing $\mathrm{MoS}_{2}$ nanosheets through $\mathrm{SnO}_{2}$ nanocrystal decoration for high-performance gas sensing in air. Small 2015, 11, 2305-2313.

156. Sarkar, D.; Xie, X.; Kang, J.; Zhang, H.; Liu, W.; Navarrete, J.; Moskovits, M.; Banerjee, K. Functionalization of transition metal dichalcogenides with metallic nanoparticles: Implications for doping and gas-sensing. Nano Lett. 2015, 15, 2852-2862.

157. Miremadi, B.; Singh, R.; Morrison, S.R.; Colbow, K. A highly sensitive and selective hydrogen gas sensor from thick oriented films of MoS 2 . Appl. Phys. A 1996, 63, 271-275.

158. Yue, Q.; Shao, Z.; Chang, S.; Li, J. Adsorption of gas molecules on monolayer $\mathrm{MoS}_{2}$ and effect of applied electric field. Nanoscale Res. Lett. 2013, 8, 1-7.

159. Zhao, S.; Xue, J.; Kang, W. Gas adsorption on $\mathrm{MoS}_{2}$ monolayer from first-principles calculations. Chem. Phys. Lett. 2014, 595-596, 35-42.

160. Cho, B.; Hahm, M.G.; Choi, M.; Yoon, J.; Kim, A.R.; Lee, Y.-J.; Park, S.-G.; Kwon, J.-D.; Kim, C.S.; Song, M.; et al. Charge-transfer-based gas sensing using atomic-layer MoS 2 . Sci. Rep. 2015, 5, 8052 .

161. Cho, B.; Yoon, J.; Lim, S.K.; Kim, A.R.; Kim, D.-H.; Park, S.-G.; Kwon, J.-D.; Lee, Y.-J.; Lee, K.-H.; Lee, B.H.; et al. Chemical sensing of $2 \mathrm{~d}$ graphene/ $\mathrm{MoS}_{2}$ heterostructure device. ACS Appl. Mater. Interfaces 2015, 7, 16775-16780.

162. Cho, B.; Kim, A.R.; Park, Y.; Yoon, J.; Lee, Y.-J.; Lee, S.; Yoo, T.J.; Kang, C.G.; Lee, B.H.; Ko, H.C.; et al. Bifunctional sensing characteristics of chemical vapor deposition synthesized atomic-layered $\mathrm{MoS}_{2}$. ACS Appl. Mater. Interfaces 2015, 7, 2952-2959.

163. Li, L.; Yu, Y.; Ye, G.J.; Ge, Q.; Ou, X.; Wu, H.; Feng, D.; Chen, X.H.; Zhang, Y. Black phosphorus field-effect transistors. Nat. Nano 2014, 9, 372-377.

164. Zhu, Z.; Tománek, D. Semiconducting layered blue phosphorus: A computational study. Phys. Rev. Lett. 2014, 112, 176802.

165. Liu, H.; Neal, A.T.; Zhu, Z.; Luo, Z.; Xu, X.; Tománek, D.; Ye, P.D. Phosphorene: An unexplored 2d semiconductor with a high hole mobility. ACS Nano 2014, 8, 4033-4041.

166. Koenig, S.P.; Doganov, R.A.; Schmidt, H.; Castro Neto, A.H.; Özyilmaz, B. Electric field effect in ultrathin black phosphorus. Appl. Phys. Lett. 2014, 104, 103106.

167. Buscema, M.; Groenendijk, D.J.; Blanter, S.I.; Steele, G.A.; van der Zant, H.S.J.; Castellanos-Gomez, A. Fast and broadband photoresponse of few-layer black phosphorus field-effect transistors. Nano Lett. 2014, 14, 3347-3352.

168. Dai, J.; Zeng, X.C. Bilayer phosphorene: Effect of stacking order on bandgap and its potential applications in thin-film solar cells. J. Phys. Chem. Lett. 2014, 5, 1289-1293.

169. Xia, F.; Wang, H.; Jia, Y. Rediscovering black phosphorus as an anisotropic layered material for optoelectronics and electronics. Nat. Commun. 2014, doi:10.1038/ncomms5458.

170. Youngblood, N.; Chen, C.; Koester, S.J.; Li, M. Waveguide-integrated black phosphorus photodetector with high responsivity and low dark current. Nat. Photonics 2015, 9, 247-252.

171. Morrison, S.R. Semiconductor gas sensors. Sens. Actuators 1981, 2, 329-341. 
172. Sun, Y.-F.; Liu, S.-B.; Meng, F.-L.; Liu, J.-Y.; Jin, Z.; Kong, L.-T.; Liu, J.-H. Metal oxide nanostructures and their gas sensing properties: A review. Sensors 2012, 12, 2610-2631.

173. Kolmakov, A.; Moskovits, M. Chemical sensing and catalysis by one-dimensional metal-oxide nanostructures. Annu. Rev. Mater. Res. 2004, 34, 151-180.

174. Brown, H.E. Zinc Oxide-Properties and Applications; International Lead Zinc Research Organization, Inc.: New York, NY, USA, 1978; p. 218.

175. Djurišić, A.B.; Ng, A.M.C.; Chen, X.Y. ZnO nanostructures for optoelectronics: Material properties and device applications. Prog. Quantum Electron. 2010, 34, 191-259.

176. Jiang, C.Y.; Sun, X.W.; Lo, G.Q.; Kwong, D.L.; Wang, J.X. Improved dye-sensitized solar cells with a ZnO-nanoflower photoanode. Appl. Phys. Lett. 2007, 90, 263501.

177. Wang, Z.L. From nanogenerators to piezotronics - A decade-long study of $\mathrm{ZnO}$ nanostructures. MRS Bull. 2012, 37, 814-827.

178. Lu, Y.; Emanetoglu, N.W.; Chen, Y. Chapter 13-ZnO piezoelectric devices. In Zinc Oxide Bulk, Thin Films and Nanostructures; Jagadish, C., Pearton, S., Eds.; Elsevier Science Ltd.: Oxford, UK, 2006; pp. 443-489.

179. Chen, Y.-J.; Zhu, C.-L.; Xiao, G. Ethanol sensing characteristics of ambient temperature sonochemically synthesized $\mathrm{ZnO}$ nanotubes. Sens. Actuators B: Chem. 2008, 129, 639-642.

180. Liao, L.; Lu, H.; Shuai, M.; Li, J.; Liu, Y.; Liu, C.; Shen, Z.; Yu, T. A novel gas sensor based on field ionization from $\mathrm{ZnO}$ nanowires: Moderate working voltage and high stability. Nanotechnology 2008, 19, 175501.

181. Heo, Y.-W.; Ren, F.; Norton, D.P. Chapter 14-Gas, chemical and biological sensing with ZnO. In Zinc Oxide Bulk, Thin Films and Nanostructures; Jagadish, C., Pearton, S., Eds.; Elsevier Science Ltd.: Oxford, UK, 2006; pp. 491-523.

182. Li, J.; Fan, H.; Jia, X. Multilayered $\mathrm{ZnO}$ nanosheets with $3 \mathrm{~d}$ porous architectures: Synthesis and gas sensing application. J. Phys. Chem. C 2010, 114, 14684-14691.

(C) 2015 by the authors; licensee MDPI, Basel, Switzerland. This article is an open access article distributed under the terms and conditions of the Creative Commons Attribution license (http://creativecommons.org/licenses/by/4.0/). 